\title{
Certain new dynamic nonlinear inequalities in two independent variables and applications
}

\author{
A.A. El-Deeb ${ }^{1 *}$ and Zareen A. Khan ${ }^{2}$
}

\section{"Correspondence:}

ahmedeldeeb@azhar.edu.eg 'Department of Mathematics, Faculty of Science, Al-Azhar University, Cairo, Egypt Full list of author information is available at the end of the article

\begin{abstract}
Several inequalities were proved in 2018 by Boudeliou, in 2015 by Abdeldain and El-Deeb and in 1998 by Pachpatte. It is our aim in this paper to generalize these inequalities to time scales. Beside that, we also apply our inequalities to discrete and continuous calculus to obtain some new inequalities as special cases. Furthermore, we study the boundedness of some problems by applying our results.
\end{abstract}

Keywords: Gronwall-type inequality; Boundedness; Time scales

\section{Introduction}

In 2018, Boudeliou [9] discussed the following inequalities.

Theorem 1.1 Suppose $a \in C\left(\hat{\Omega}, \mathbb{R}_{+}\right)$is nondecreasing with respect to $(\breve{x}, \breve{y}) \in \hat{\Omega}=I_{1} \times I_{2}$, let $\hat{\alpha}(\breve{x}) \in C^{1}\left(I_{1}, I_{2}\right)$ and $\hat{\beta}(\breve{y}) \in C^{1}\left(I_{2}, I_{2}\right)$ be nondecreasing functions with $\hat{\alpha}(\breve{x}) \leq \breve{x}$ on $I_{1}, \hat{\beta}(\breve{y}) \leq$ $\breve{y}$, and $g, u, p, f \in C\left(\hat{\Omega}, \mathbb{R}_{+}\right)$. Furthermore, suppose $\bar{\psi}, \bar{\varphi} \in C\left(\mathbb{R}_{+}, \mathbb{R}_{+}\right)$are nondecreasing functions with $\{\bar{\psi}, \bar{\varphi}\}(u)>0$ for $u>0$, and $\lim _{u \rightarrow+\infty} \bar{\psi}(u)=+\infty$. If $u(\breve{x}, \breve{y})$ satisfies

$$
\begin{aligned}
\bar{\psi}(u(\breve{x}, \breve{y})) \leq & a(\breve{x}, \breve{y})+\int_{0}^{\hat{\alpha}(\breve{x})} \int_{0}^{\hat{\beta}(\breve{y})}[f(\breve{s}, \breve{t}) \bar{\varphi}(u(\breve{s}, \breve{t}))+p(\breve{s}, \breve{t})] d \breve{t} d \breve{s} \\
& +\int_{0}^{\hat{\alpha}(\breve{x})} \int_{0}^{\hat{\beta}(\breve{y})} f(\breve{s}, \breve{t})\left(\int_{0}^{\breve{s}} g(\breve{\tau}, \breve{t}) \bar{\varphi}(u(\breve{\tau}, \breve{t})) d \breve{\tau}\right) d \breve{t} d \breve{s},
\end{aligned}
$$

for $(\breve{x}, \breve{y}) \in \hat{\Omega}$, then

$$
u(\breve{x}, \breve{y}) \leq \bar{\psi}^{-1}\left\{\breve{G}^{-1}\left[\breve{G}(q(\breve{x}, \breve{y}))+\int_{0}^{\hat{\alpha}(\breve{x})} \int_{0}^{\hat{\beta}(\breve{y})} f(\breve{s}, \breve{t})\left(1+\int_{0}^{\breve{s}} g(\breve{\tau}, \breve{t}) d \breve{\tau}\right) d \breve{t} d \breve{s}\right]\right\}
$$

for $0 \leq \breve{x} \leq \breve{x}_{1}, 0 \leq \breve{y} \leq \breve{y}_{1}$, where

$$
q(\breve{x}, \breve{y})=a(\breve{x}, \breve{y})+\int_{0}^{\hat{\alpha}(\breve{x})} \int_{0}^{\hat{\beta}(\breve{y})} p(\breve{s}, \breve{t}) d \breve{t} d \breve{s},
$$

(c) The Author(s) 2020. This article is licensed under a Creative Commons Attribution 4.0 International License, which permits use, sharing, adaptation, distribution and reproduction in any medium or format, as long as you give appropriate credit to the original author(s) and the source, provide a link to the Creative Commons licence, and indicate if changes were made. The images or other third party material in this article are included in the article's Creative Commons licence, unless indicated otherwise in a credit line to the material. If material is not included in the article's Creative Commons licence and your intended use is not permitted by statutory regulation or exceeds the permitted use, you will need to obtain permission directly from the copyright holder. To view a copy of this licence, visit http://creativecommons.org/licenses/by/4.0/. 


$$
\breve{G}(r)=\int_{r_{0}}^{r} \frac{d \breve{s}}{\bar{\varphi} \circ \bar{\psi}^{-1}(\breve{s})}, \quad r \geq r_{0}>0, \quad \breve{G}(+\infty)=\int_{r_{0}}^{+\infty} \frac{d \breve{s}}{\bar{\varphi} \circ \bar{\psi}^{-1}(\breve{s})}=+\infty,
$$

and $\left(\breve{x}_{1}, \breve{y}_{1}\right) \in \hat{\Omega}$ is chosen so that

$$
\left(\breve{G}(q(\breve{x}, \breve{y}))+\int_{0}^{\hat{\alpha}(\breve{x})} \int_{0}^{\hat{\beta}(\breve{y})} f(\breve{s}, \breve{t})\left(1+\int_{0}^{\breve{s}} g(\breve{\tau}, \breve{t}) d \breve{\tau}\right) d \breve{t} d \breve{s}\right) \in \operatorname{Dom}\left(G^{-1}\right)
$$

Theorem 1.2 Assume that $g, a, f, u, \hat{\beta}, \hat{\alpha},, \bar{\psi}$ and $\bar{\varphi}$ be as in Theorem 1.1. If $u(\breve{x}, \breve{y})$ satisfies

$$
\begin{aligned}
\bar{\psi}(u(\breve{x}, \breve{y})) \leq & a(\breve{x}, \breve{y})+\left(\int_{0}^{\hat{\alpha}(\breve{x})} \int_{0}^{\hat{\beta}(\breve{y})} f(\breve{s}, \breve{t}) \bar{\varphi}(u(\breve{s}, \breve{t})) d \breve{t} d \breve{s}\right)^{2} \\
& +\int_{0}^{\hat{\alpha}(\breve{x})} \int_{0}^{\hat{\beta}(\breve{y})} f(\breve{s}, \breve{t}) \bar{\varphi}(u(\breve{s}, \breve{t}))\left(\int_{0}^{\breve{s}} g(\breve{t}, \breve{t}) \bar{\varphi}(u(\breve{\tau}, \breve{t})) d \breve{\tau}\right) d \breve{t} d \breve{s},
\end{aligned}
$$

for $(\breve{x}, \breve{y}) \in \hat{\Omega}$, then

$$
u(\breve{x}, \breve{y}) \leq \bar{\psi}^{-1}\left\{\breve{H}^{-1}\left[\breve{H}(a(\breve{x}, \breve{y}))+\breve{B}(\breve{x}, \breve{y})+\left(\int_{0}^{\hat{\alpha}(\breve{x})} \int_{0}^{\hat{\beta}(\breve{y})} f(\breve{s}, \breve{t}) d \breve{t} d \breve{s}\right)^{2}\right]\right\}
$$

for $0 \leq \breve{x} \leq \breve{x}_{1}, 0 \leq \breve{y} \leq \breve{y}_{1}$, where

$$
\begin{aligned}
& \breve{B}(\breve{x}, \breve{y})=\int_{0}^{\hat{\alpha}(\breve{x})} \int_{0}^{\hat{\beta}(\breve{y})} f(\breve{s}, \breve{t})\left(\int_{0}^{\breve{s}} g(\breve{\tau}, \breve{t}) d \breve{\tau}\right) d \breve{t} d \breve{s}, \\
& \breve{H}(r)=\int_{r_{0}}^{r} \frac{d \breve{s}}{\left(\bar{\varphi} \circ \bar{\psi}^{-1}\right)^{2}(\breve{s})}, \quad r \geq r_{0}>0, \quad \breve{H}(+\infty)=\int_{r_{0}}^{+\infty} \frac{d \breve{s}}{\left(\bar{\varphi} \circ \bar{\psi}^{-1}\right)^{2}(\breve{s})}=+\infty,
\end{aligned}
$$

and $\left(\breve{x}_{1}, \breve{y}_{1}\right) \in \hat{\Omega}$ is chosen so that

$$
\left(\breve{H}(a(\breve{x}, \breve{y}))+B(\breve{x}, \breve{y})+\left(\int_{0}^{\hat{\alpha}(\breve{x})} \int_{0}^{\hat{\beta}(\breve{y})} f(\breve{s}, \breve{t}) d \breve{t} d \breve{s}\right)^{2}\right) \in \operatorname{Dom}\left(\breve{H}^{-1}\right) .
$$

In 1988, Hilger [33] presented time scale theory to unify continuous and discrete analysis. For some Gronwall-Bellman-type integral, dynamic inequalities and other type inequalities on time scales, see Refs. [1-8, 13, 14, 16-32, 34-41]. For more details on time scales calculus see [15].

A time scale $\mathbb{T}$ is an arbitrary nonempty closed subset of $\mathbb{R}$. We suppose throughout the article that $\mathbb{T}$ has the topology that it inherits from the standard topology on $\mathbb{R}$. The forward jump operator $\sigma: \mathbb{T} \rightarrow \mathbb{T}$ is defined for any $t \in \mathbb{T}$ by

$$
\sigma(t):=\inf \{s \in \mathbb{T}: s>t\}
$$

and the backward jump operator $\rho: \mathbb{T} \rightarrow \mathbb{T}$ is defined for any $t \in \mathbb{T}$ by

$$
\rho(t):=\sup \{s \in \mathbb{T}: s<t\}
$$

In the previous two definitions, we set $\inf \emptyset=\sup \mathbb{T}$ (i.e., if $t$ is the maximum of $\mathbb{T}$, then $\sigma(t)=t$ ) and $\sup \emptyset=\inf \mathbb{T}$ (i.e., if $t$ is the minimum of $\mathbb{T}$, then $\rho(t)=t$ ), where $\emptyset$ is the empty set. 
A point $t \in \mathbb{T}$ with $\inf \mathbb{T}<t<\sup \mathbb{T}$ is said to be right-scattered if $\sigma(t)>t$, right-dense if $\sigma(t)=t$, left-scattered if $\rho(t)<t$, and left-dense if $\rho(t)=t$. Points that are simultaneously right-dense and left-dense are called dense points. Points that are simultaneously rightscattered and left-scattered are called isolated points.

We define the forward graininess function $\mu: \mathbb{T} \rightarrow[0, \infty)$ for any $t \in \mathbb{T}$ by $\mu(t):=\sigma(t)-t$.

Let $f: \mathbb{T} \rightarrow \mathbb{R}$ be a function. Then the function $f^{\sigma}: \mathbb{T} \rightarrow \mathbb{R}$ is defined by $f^{\sigma}(t)=f(\sigma(t))$, $\forall t \in \mathbb{T}$, that is, $f^{\sigma}=f \circ \sigma$. In a similar manner, the function $f^{\rho}: \mathbb{T} \rightarrow \mathbb{R}$ is defined by $f^{\rho}(t)=f(\rho(t)), \forall t \in \mathbb{T}$, that is, $f^{\rho}=f \circ \rho$.

We introduce the set $\mathbb{T}^{\kappa}$ as follows: If $\mathbb{T}$ has a left-scattered maximum $m$, then $\mathbb{T}^{\kappa}=$ $\mathbb{T}-\{m\}$, otherwise $\mathbb{T}^{\kappa}=\mathbb{T}$.

The interval $[a, b]$ in $\mathbb{T}$ is defined by

$$
[a, b]_{\mathbb{T}}=\{t \in \mathbb{T}: a \leq t \leq b\}
$$

Open intervals and half-closed interval are defined similarly.

Suppose $f: \mathbb{T} \rightarrow \mathbb{R}$ is a function and $t \in \mathbb{T}^{\kappa}$. Then we say that $f^{\Delta}(t) \in \mathbb{R}$ is the delta derivative of $f$ at $t$ if for any $\varepsilon>0$ there exists a neighborhood $U$ of $t$ such that, for all $s \in U$, we have

$$
\left|[f(\sigma(t))-f(s)]-f^{\Delta}(t)[\sigma(t)-s]\right| \leq \varepsilon|\sigma(t)-s|
$$

Furthermore, $f$ is said to be delta differentiable on $\mathbb{T}^{\kappa}$ if it is delta differentiable at each $t \in \mathbb{T}^{\kappa}$.

If $f, g: \mathbb{T} \rightarrow \mathbb{R}$ are delta differentiable functions at $t \in \mathbb{T}^{\kappa}$, then

1. $(f+g)^{\Delta}(t)=f^{\Delta}(t)+g^{\Delta}(t)$

2. $(f g)^{\Delta}(t)=f^{\Delta}(t) g(t)+f(\sigma(t)) g^{\Delta}(t)=f(t) g^{\Delta}(t)+f^{\Delta}(t) g(\sigma(t))$;

3. $\left(\frac{f}{g}\right)^{\Delta}(t)=\frac{f^{\Delta}(t) g(t)-f(t) g^{\Delta}(t)}{g(t) g(\sigma(t))}$ provided $g(t) g(\sigma(t)) \neq 0$.

A function $g: \mathbb{T} \rightarrow \mathbb{R}$ is called right-dense continuous (rd-continuous) if $g$ is continuous at the right-dense points in $\mathbb{T}$ and its left-sided limits exist at all left-dense points in $\mathbb{T}$.

A function $F: \mathbb{T} \rightarrow \mathbb{R}$ is said to be a delta antiderivative of $f: \mathbb{T} \rightarrow \mathbb{R}$ if $F^{\Delta}(t)=f(t)$ for all $t \in \mathbb{T}^{\kappa}$. In this case, the definite delta integral of $f$ is defined by

$$
\int_{a}^{b} f(\eta) \Delta \eta=F(b)-F(a) \quad \text { for all } a, b \in \mathbb{T} \text {. }
$$

If $g \in C_{\mathrm{rd}}(\mathbb{T})$ and $t, t_{0} \in \mathbb{T}$, then the definite integral $G(t):=\int_{t_{0}}^{t} g(s) \Delta s$ exists, and $G^{\Delta}(t)=$ $g(t)$ holds.

Assume that $a, b, c \in \mathbb{T}, \alpha \in \mathbb{R}$, and $f, g$ be continuous functions on $[a, b]_{\mathbb{T}}$. Then

1. $\int_{a}^{b}[f(t)+g(t)] \Delta \eta=\int_{a}^{b} f(\eta) \Delta \eta+\int_{a}^{b} g(\eta) \Delta \eta$;

2. $\int_{a}^{b} \alpha f(\eta) \Delta \eta=\alpha \int_{a}^{b} f(\eta) \Delta \eta$

3. $\int_{a}^{b} f(\eta) \Delta \eta=\int_{a}^{c} f(\eta) \Delta \eta+\int_{c}^{b} f(\eta) \Delta \eta$;

4. $\int_{a}^{b} f(\eta) \Delta \eta=-\int_{b}^{a} f(\eta) \Delta \eta$

5. $\int_{a}^{a} f(\eta) \Delta \eta=0$;

6. if $f(t) \geq g(t)$ on $[a, b]_{\mathbb{T}}$, then $\int_{a}^{b} f(\eta) \Delta \eta \geq \int_{a}^{b} g(\eta) \Delta \eta$.

We will need the following important relations between calculus on time scales $\mathbb{T}$ and either continuous calculus on $\mathbb{R}$ or discrete calculus on $\mathbb{Z}$. Note that: 
1. If $\mathbb{T}=\mathbb{R}$, then

$$
\sigma(t)=t, \quad \mu(t)=0, \quad f^{\Delta}(t)=f^{\prime}(t), \quad \int_{a}^{b} f(\eta) \Delta \eta=\int_{a}^{b} f(t) d t .
$$

2. If $\mathbb{T}=\mathbb{Z}$, then

$$
\sigma(t)=t+1, \quad \mu(t)=1, \quad f^{\Delta}(t)=f(t+1)-f(t), \quad \int_{a}^{b} f(\eta) \Delta \eta=\sum_{t=a}^{b-1} f(t) .
$$

In the following, we present the basic theorems that will be needed in the proofs of our main results.

Theorem 1.3 If $\hat{f}$ is $\hat{\Delta}$-integrable on $[a, b]$, then so is $|\hat{f}|$, and

$$
\left|\int_{a}^{b} \hat{f}(\check{t}) \hat{\Delta} \check{t}\right| \leq \int_{a}^{b}|\hat{f}(\check{t})| \hat{\Delta} \check{t}
$$

Theorem 1.4 (Chain rule on time scales [15]) Assume $\hat{g}: \mathbb{R} \rightarrow \mathbb{R}$ is continuous, $\hat{g}: \breve{\mathbb{T}} \rightarrow \mathbb{R}$ is $\hat{\Delta}$-differentiable on $\mathbb{T}^{\kappa}$, and $\hat{f}: \mathbb{R} \rightarrow \mathbb{R}$ is continuously differentiable. Then there exists $c \in[\check{t}, \sigma(\check{t})]$ with

$$
(\hat{f} \circ \hat{g})^{\hat{\Delta}}(\check{t})=\hat{f}^{\prime}(\hat{g}(c)) \hat{g}^{\hat{\Delta}}(\check{t}) .
$$

Theorem 1.5 (Chain rule on time scales [15]) Let $\hat{f}: \mathbb{R} \rightarrow \mathbb{R}$ be continuously differentiable and suppose $\hat{g}: \breve{\mathbb{T}} \rightarrow \mathbb{R}$ is $\hat{\Delta}$-differentiable. Then $f \circ \hat{g}: \breve{\mathbb{T}} \rightarrow \mathbb{R}$ is $\hat{\Delta}$-differentiable and the formula

$$
(\hat{f} \circ \hat{g})^{\hat{\Delta}}(\check{t})=\left\{\int_{0}^{1}\left[\hat{f}^{\prime}\left(h \hat{g}^{\sigma}(\check{t})+(1-h) \hat{g}(\check{t})\right)\right] d h\right\} \hat{g}^{\hat{\hat{\Delta}}}(\check{t})
$$

holds.

This paper gives us the time scale versions of the results provided in [9]. These inequalities, proved here, extend some known results in the literature, and they are also unify the continuous and the discrete case.

\section{Main results}

In what follows, $\mathbb{R}$ denotes the set of real numbers, $\mathbb{R}_{+}=[0,+\infty), \breve{\mathbb{T}}_{1}, \breve{\mathbb{T}}_{2}$ are two time scales and we put $\Omega=\breve{\mathbb{T}}_{1} \times \breve{\mathbb{T}}_{2}=\left\{(\breve{t}, \breve{s}): \breve{t} \in \breve{\mathbb{T}}_{1}, \breve{s} \in \breve{\mathbb{T}}_{2}\right\}$ which is a complete metric space with the metric $\breve{\rho}$ defined by

$$
\breve{\rho}((\breve{t}, \breve{s}),(\dot{t}, \hat{s}))=\sqrt{(\breve{t}-\dot{t})^{2}+(\breve{s}-\hat{s})^{2}}, \quad \forall(\breve{t}, \breve{s}),(\dot{t}, \hat{s}) \in \breve{\mathbb{T}}_{1} \times \breve{\mathbb{T}}_{2} .
$$

$C_{\mathrm{rd}}\left(\Omega, \mathbb{R}_{+}\right)$denotes the set of all right-dense continuous functions from $\Omega$ into $\mathbb{R}_{+}$and $C_{\mathrm{rd}}^{1}\left(\breve{\mathbb{T}}_{i}, \breve{\mathbb{T}}_{i}\right)$ denotes the set of all right-dense continuously delta-differentiable functions from $\breve{\mathbb{T}}_{i}$ into $\breve{\mathbb{T}}_{i}, i=1,2$. The two-variables time scales calculus and multiple integration on time scales were introduced in $[10,11]$ (see also [12]). 
Theorem 2.1 Suppose that $a \in C_{\mathrm{rd}}\left(\Omega, \mathbb{R}_{+}\right)$is nondecreasing with respect to $(\breve{x}, \breve{y}) \in \Omega$, and $g, u, p, f \in C_{\mathrm{rd}}\left(\Omega, \mathbb{R}_{+}\right)$. Furthermore, suppose that $\bar{\psi}, \bar{\varphi} \in C\left(\mathbb{R}_{+}, \mathbb{R}_{+}\right)$are nondecreasing functions with $\{\bar{\psi}, \bar{\varphi}\}(u)>0$ for $u>0$, and $\lim _{u \rightarrow+\infty} \bar{\psi}(u)=+\infty$. If $u(\breve{x}, \breve{y})$ satisfies

$$
\begin{aligned}
\bar{\psi}(u(\breve{x}, \breve{y})) \leq & a(\breve{x}, \breve{y})+\int_{0}^{\breve{x}} \int_{0}^{\breve{y}}[f(\breve{s}, \breve{t}) \bar{\varphi}(u(\breve{s}, \breve{t}))+p(\breve{s}, \breve{t})] \hat{\Delta} \breve{t} \hat{\Delta} \breve{s} \\
& +\int_{0}^{\breve{x}} \int_{0}^{\check{y}} f(\breve{s}, \breve{t})\left(\int_{0}^{\breve{s}} g(\breve{\tau}, \breve{t}) \bar{\varphi}(u(\breve{\tau}, \breve{t})) \hat{\Delta} \breve{\tau}\right) \hat{\Delta} \breve{t} \hat{\Delta} \breve{s},
\end{aligned}
$$

for $(\breve{x}, \breve{y}) \in \Omega$, then

$$
u(\breve{x}, \breve{y}) \leq \bar{\psi}^{-1}\left\{\breve{G}^{-1}\left[\breve{G}(q(\breve{x}, \breve{y}))+\int_{0}^{\breve{x}} \int_{0}^{\breve{y}} f(\breve{s}, \breve{t})\left(1+\int_{0}^{\breve{s}} g(\breve{\tau}, \breve{t}) \hat{\Delta} \breve{\tau}\right) \hat{\Delta} \breve{t} \hat{\Delta} \breve{s}\right]\right\}
$$

for $0 \leq \breve{x} \leq \breve{x}_{1}, 0 \leq \breve{y} \leq \breve{y}_{1}$, where

$$
\begin{aligned}
& q(\breve{x}, \breve{y})=a(\breve{x}, \breve{y})+\int_{0}^{\breve{x}} \int_{0}^{\breve{y}} p(\breve{s}, \breve{t}) \hat{\Delta} \breve{t} \hat{\Delta} \breve{s}, \\
& \breve{G}(r)=\int_{r_{0}}^{r} \frac{\hat{\Delta} \breve{\varphi}}{\bar{\varphi} \circ \bar{\psi}^{-1}(\breve{s})}, \quad r \geq r_{0}>0, \quad \breve{G}(+\infty)=\int_{r_{0}}^{+\infty} \frac{\hat{\Delta} \breve{s}}{\bar{\varphi} \circ \bar{\psi}^{-1}(\breve{s})}=+\infty,
\end{aligned}
$$

and $\left(\breve{x}_{1}, \breve{y}_{1}\right) \in \Omega$ is chosen so that

$$
\left(\breve{G}(q(\breve{x}, \breve{y}))+\int_{0}^{\breve{x}} \int_{0}^{\breve{y}} f(\breve{s}, \breve{t})\left(1+\int_{0}^{\breve{s}} g(\breve{\tau}, \breve{t}) \hat{\Delta} \breve{\tau}\right) \hat{\Delta} \breve{t} \hat{\Delta} \breve{s}\right) \in \operatorname{Dom}\left(G^{-1}\right) .
$$

Proof Assume that $a(\breve{x}, \breve{y})>0$. Since $q \geq 0$ and it is nondecreasing, fixing an arbitrary point $(\breve{\xi}, \breve{\zeta}) \in \Omega$ and defining $z(\breve{x}, \breve{y})$ by

$$
\begin{aligned}
z(\breve{x}, \breve{y})= & q(\breve{\xi}, \breve{\zeta})+\int_{0}^{\breve{x}} \int_{0}^{\breve{y}} f(\breve{s}, \breve{t}) \bar{\varphi}(u(\breve{s}, \breve{t})) \hat{\Delta} \breve{t} \hat{\Delta} \breve{s} \\
& +\int_{0}^{\breve{x}} \int_{0}^{\breve{y}} f(\breve{s}, \breve{t})\left(\int_{0}^{\breve{s}} g(\breve{\tau}, \breve{t}) \bar{\varphi}(u(\breve{\tau}, \breve{t})) \hat{\Delta} \breve{\tau}\right) \hat{\Delta} \breve{t} \hat{\Delta} \breve{s},
\end{aligned}
$$

which is a positive and nondecreasing function for $0 \leq \breve{x} \leq \breve{\xi} \leq \breve{x}_{1}, 0 \leq \breve{x} \leq \breve{\zeta} \leq \breve{y}_{1}$, we have $z(0, \breve{y})=z(\breve{x}, 0)=q(\breve{\xi}, \breve{\zeta})$ and

$$
u(\breve{x}, \breve{y}) \leq \bar{\psi}^{-1}(z(\breve{x}, \breve{y}))
$$

Differentiating $z(\breve{x}, \breve{y})$, with respect to $\breve{x}$ and using (2.4), we get

$$
\begin{aligned}
z^{\hat{\Delta} \breve{x}}(\breve{x}, \breve{y}) & =\int_{0}^{\breve{y}} f(\breve{x}, \breve{t})\left[\bar{\varphi}(u(\breve{x}, \breve{t}))+\int_{0}^{\breve{x}} g(\breve{\tau}, \breve{t}) \bar{\varphi}(u(\breve{\tau}, \breve{t})) \hat{\Delta} \breve{\tau}\right] \hat{\Delta} \breve{t} \\
& \leq \int_{0}^{\breve{y}} f(\breve{x}, \breve{t})\left[\bar{\varphi} \circ \bar{\psi}^{-1}(z(\breve{x}, \breve{t}))+\int_{0}^{\breve{x}} g(\breve{\tau}, \breve{t}) \bar{\varphi} \circ \bar{\psi}^{-1}(z(\breve{\tau}, \breve{t})) \hat{\Delta} \breve{\tau}\right] \hat{\Delta} \breve{t}
\end{aligned}
$$


since $\bar{\varphi} \circ \bar{\psi}^{-1}$ is nondecreasing with respect to $(\breve{x}, \breve{y}) \in \mathbb{R}_{+} \times \mathbb{R}_{+}$, we have

$$
\begin{aligned}
z^{\hat{\Delta} \breve{x}}(\breve{x}, \breve{y}) & \leq \int_{0}^{\breve{y}} f(\breve{x}, \breve{t})\left[\bar{\varphi} \circ \bar{\psi}^{-1}(z(\breve{x}, \breve{t}))+\bar{\varphi} \circ \bar{\psi}^{-1}(z(\breve{x}, \breve{t})) \int_{0}^{\breve{x}} g(\breve{t}, \breve{t}) \hat{\Delta} \breve{\tau}\right] \hat{\Delta} \breve{t} \\
& \leq \bar{\varphi} \circ \bar{\psi}^{-1}(z(\breve{x}, \breve{y})) \int_{0}^{\breve{y}} f(\breve{x}, \breve{t})\left[1+\int_{0}^{\breve{x}} g(\breve{\tau}, \breve{t}) \hat{\Delta} \breve{\tau}\right] \hat{\Delta} \breve{t},
\end{aligned}
$$

and from (2.5) we get

$$
\frac{z^{\hat{\Delta} \breve{x}(\breve{x}, \breve{y})}}{\bar{\varphi} \circ \bar{\psi}^{-1}(z(\breve{x}, \breve{y}))} \leq \int_{0}^{\breve{y}} f(\breve{x}, \breve{t})\left(1+\int_{0}^{\breve{x}} g(\breve{\tau}, \breve{t}) \hat{\Delta} \breve{\tau}\right) \hat{\Delta} \breve{t} .
$$

From (2.6) we get

$$
\breve{G}(z(\breve{x}, \breve{y})) \leq \breve{G}(q(\breve{\xi}, \breve{\zeta}))+\int_{0}^{\breve{x}} \int_{0}^{\breve{y}} f(\breve{s}, \breve{t})\left(1+\int_{0}^{\breve{s}} g(\breve{\tau}, \breve{t}) \hat{\Delta} \breve{\tau}\right) \hat{\Delta} \breve{t} \hat{\Delta} \breve{s} .
$$

Since $(\breve{\xi}, \breve{\zeta}) \in \Omega$ is chosen arbitrarily,

$$
z(\breve{x}, \breve{y}) \leq \breve{G}^{-1}\left[\breve{G}(q(\breve{x}, \breve{y}))+\int_{0}^{\breve{x}} \int_{0}^{\breve{y}} f(\breve{s}, \breve{t})\left(1+\int_{0}^{\breve{s}} g(\breve{\tau}, \breve{t}) \hat{\Delta} \breve{\tau}\right) \hat{\Delta} \breve{t} \hat{\Delta} \breve{s}\right] .
$$

So from (2.7) and (2.4) we get the desired inequality in (2.1). For $a(\breve{x}, \breve{y})=0$, we carry out the above procedure with $\epsilon>0$ instead of $a(\breve{x}, \breve{y})$ and subsequently let $\epsilon \rightarrow 0$. This completes the proof.

Corollary 2.2 If we take $\breve{\mathbb{T}}=\mathbb{R}$ in Theorem 2.1 , then the inequality

$$
\begin{aligned}
\bar{\psi}(u(\breve{x}, \breve{y})) \leq & a(\breve{x}, \breve{y})+\int_{0}^{\breve{x}} \int_{0}^{\breve{y}}[f(\breve{s}, \breve{t}) \bar{\varphi}(u(\breve{s}, \breve{t}))+p(\breve{s}, \breve{t})] d \breve{t} d \breve{s} \\
& +\int_{0}^{\breve{x}} \int_{0}^{\breve{y}} f(\breve{s}, \breve{t})\left(\int_{0}^{\breve{s}} g(\breve{\tau}, \breve{t}) \bar{\varphi}(u(\breve{\tau}, \breve{t})) d \breve{\tau}\right) d \breve{t} d \breve{s},
\end{aligned}
$$

for $(\breve{x}, \breve{y}) \in \Omega$, implies

$$
u(\breve{x}, \breve{y}) \leq \bar{\psi}^{-1}\left\{\breve{G}^{-1}\left[\breve{G}(q(\breve{x}, \breve{y}))+\int_{0}^{\breve{x}} \int_{0}^{\breve{y}} f(\breve{s}, \breve{t})\left(1+\int_{0}^{\breve{s}} g(\breve{\tau}, \breve{t}) d \breve{\tau}\right) d \breve{t} d \breve{s}\right]\right\}
$$

for $0 \leq \breve{x} \leq \breve{x}_{1}, 0 \leq \breve{y} \leq \breve{y}_{1}$, where

$$
\begin{aligned}
& q(\breve{x}, \breve{y})=a(\breve{x}, \breve{y})+\int_{0}^{\breve{x}} \int_{0}^{\breve{y}} p(\breve{s}, \breve{t}) d \breve{t} d \breve{s}, \\
& \breve{G}(r)=\int_{r_{0}}^{r} \frac{d \breve{s}}{\bar{\varphi} \circ \bar{\psi}^{-1}(\breve{s})}, \quad r \geq r_{0}>0, \quad \breve{G}(+\infty)=\int_{r_{0}}^{+\infty} \frac{d \breve{s}}{\bar{\varphi} \circ \bar{\psi}^{-1}(\breve{s})}=+\infty,
\end{aligned}
$$

and $\left(\breve{x}_{1}, \breve{y}_{1}\right) \in \Omega$ is chosen so that

$$
\left(\breve{G}(q(\breve{x}, \breve{y}))+\int_{0}^{\breve{x}} \int_{0}^{\breve{y}} f(\breve{s}, \breve{t})\left(1+\int_{0}^{\breve{s}} g(\breve{\tau}, \breve{t}) d \breve{\tau}\right) d \breve{t} d \breve{s}\right) \in \operatorname{Dom}\left(G^{-1}\right) .
$$


Corollary 2.3 The discrete form can be obtained by letting $\breve{\mathbb{T}}=\mathbb{Z}$ in Theorem 2.1:

$$
\begin{aligned}
\bar{\psi}(u(\breve{x}, \breve{y})) \leq & a(\breve{x}, \breve{y})+\sum_{\breve{s}=0}^{\breve{x}-1} \sum_{\breve{t}=0}^{\breve{y}-1}[f(\breve{s}, \breve{t}) \bar{\varphi}(u(\breve{s}, \breve{t}))+p(\breve{s}, \breve{t})] \\
& +\sum_{\breve{s}=0}^{\breve{x}-1} \sum_{\breve{t}=0}^{\breve{y}-1} f(\breve{s}, \breve{t})\left(\sum_{\breve{t}=0}^{\breve{s}-1} g(\breve{\tau}, \breve{t}) \bar{\varphi}(u(\breve{\tau}, \breve{t}))\right)
\end{aligned}
$$

for $(\breve{x}, \breve{y}) \in \Omega$, which implies

$$
u(\breve{x}, \breve{y}) \leq \bar{\psi}^{-1}\left\{\breve{G}^{-1}\left[\breve{G}(q(\breve{x}, \breve{y}))+\sum_{\breve{s}=0}^{\breve{x}-1} \sum_{\breve{t}=0}^{\breve{y}-1} f(\breve{s}, \breve{t})\left(1+\sum_{\breve{\tau}=0}^{\breve{s}-1} g(\breve{t}, \breve{t})\right)\right]\right\}
$$

for $0 \leq \breve{x} \leq \breve{x}_{1}, 0 \leq \breve{y} \leq \breve{y}_{1}$, where

$$
\begin{aligned}
& q(\breve{x}, \breve{y})=a(\breve{x}, \breve{y})+\sum_{\breve{s}=0}^{\breve{x}-1} \sum_{\breve{t}=0}^{\breve{y}-1} p(\breve{s}, \breve{t}), \\
& \breve{G}(r)=\sum_{\breve{s}=r_{0}}^{r-1} \frac{1}{\bar{\varphi} \circ \bar{\psi}^{-1}(\breve{s})}, \quad r \geq r_{0}>0, \quad \breve{G}(+\infty)=\sum_{\breve{s}=r_{0}}^{+\infty} \frac{1}{\bar{\varphi} \circ \bar{\psi}^{-1}(\breve{s})}=+\infty,
\end{aligned}
$$

and $\left(\breve{x}_{1}, \breve{y}_{1}\right) \in \Omega$ is chosen so that

$$
\left(\breve{G}(q(\breve{x}, \breve{y}))+\sum_{\breve{s}=0}^{\breve{x}-1} \sum_{\breve{t}=0}^{\breve{y}-1} f(\breve{s}, \breve{t})\left(1+\sum_{\breve{\tau}_{0}}^{\breve{s}} g(\breve{\tau}, \breve{t})\right)\right) \in \operatorname{Dom}\left(G^{-1}\right) .
$$

Theorem 2.4 Assume that $h, b \in C_{\mathrm{rd}}\left(\Omega, \mathbb{R}_{+}\right)$. Let $g, f, p, a, u, \bar{\psi}$ and $\bar{\varphi}$ be as in Theorem 2.1, if $u(\breve{x}, \breve{y})$ satisfies

$$
\begin{aligned}
\bar{\psi}(u(\breve{x}, \breve{y})) \leq & a(\breve{x}, \breve{y})+\int_{0}^{\breve{x}} \int_{0}^{\breve{y}}[f(\breve{s}, \breve{t}) \bar{\varphi}(u(\breve{s}, \breve{t}))+p(\breve{s}, \breve{t})] \hat{\Delta} \breve{t} \hat{\Delta} \breve{s} \\
& +\int_{0}^{\breve{x}} \int_{0}^{\breve{y}} b(\breve{s}, \breve{t})\left[h(\breve{s}, \breve{t}) \bar{\varphi}(u(\breve{s}, \breve{t}))+\int_{0}^{\breve{s}} g(\breve{\tau}, \breve{t}) \bar{\varphi}(u(\breve{\tau}, \breve{t})) \hat{\Delta} \breve{\tau}\right] \hat{\Delta} \breve{t} \hat{\Delta} \breve{s}
\end{aligned}
$$

for $(\breve{x}, \breve{y}) \in \Omega$, then

$$
u(\breve{x}, \breve{y}) \leq \bar{\psi}^{-1}\left\{\breve{G}^{-1}\left[\breve{G}(q(\breve{x}, \breve{y}))+A(\breve{x}, \breve{y})+\int_{0}^{\breve{x}} \int_{0}^{\breve{y}} f(\breve{s}, \breve{t}) \hat{\Delta} \breve{t} \hat{\Delta} \breve{s}\right]\right\}
$$

for $0 \leq \breve{x} \leq \breve{x}_{1}, 0 \leq \breve{y} \leq \breve{y}_{1}$, where $q, \breve{G}$ are defined by (2.2) and (2.3), respectively, and

$$
\breve{A}(\breve{x}, \breve{y})=\int_{0}^{\breve{x}} \int_{0}^{\breve{y}} b(\breve{s}, \breve{t})\left[h(\breve{s}, \breve{t})+\int_{0}^{\breve{s}} g(\breve{\tau}, \breve{t}) \hat{\Delta} \breve{\tau}\right] \hat{\Delta} \breve{t} \hat{\Delta} \breve{s}
$$

and $\left(\breve{x}_{1}, \breve{y}_{1}\right) \in \Omega$ is chosen so that

$$
\left(\breve{G}(q(\breve{x}, \breve{y}))+\breve{A}(\breve{x}, \breve{y})+\int_{0}^{\breve{x}} \int_{0}^{\breve{y}} f(\breve{s}, \breve{t}) \hat{\Delta} \breve{t} \hat{\Delta} \breve{s}\right) \in \operatorname{Dom}\left(\breve{G}^{-1}\right) .
$$


Proof Assume that $a(\breve{x}, \breve{y})>0$. Fixing an arbitrary $(\breve{\xi}, \breve{\zeta}) \in \Omega$, we define positive and nondecreasing function $z(\breve{x}, \breve{y})$ by

$$
\begin{aligned}
z(\breve{x}, \breve{y})= & q(\breve{\xi}, \breve{\zeta})+\int_{0}^{\breve{x}} \int_{0}^{\breve{y}} f(\breve{s}, \breve{t}) \bar{\varphi}(u(\breve{s}, \breve{t})) \hat{\Delta} \breve{t} \hat{\Delta} \breve{s} \\
& +\int_{0}^{\breve{x}} \int_{0}^{\breve{y}} b(\breve{s}, \breve{t})\left[h(\breve{s}, \breve{t}) \bar{\varphi}(u(\breve{s}, \breve{t}))+\int_{0}^{\breve{s}} g(\breve{t}, \breve{t}) \bar{\varphi}(u(\breve{t}, \breve{t})) \hat{\Delta} \breve{\tau}\right] \hat{\Delta} \breve{t} \hat{\Delta} \breve{s}
\end{aligned}
$$

for $0 \leq \breve{x} \leq \breve{\xi} \leq \breve{x}_{1}, 0 \leq \breve{y} \leq \breve{\zeta} \leq y_{1}$, then $z(0, \breve{y})=z(\breve{x}, 0)=q(\breve{\xi}, \breve{\zeta})$ and

$$
u(\breve{x}, \breve{y}) \leq \bar{\psi}^{-1}(z(\breve{x}, \breve{y}))
$$

then we have

$$
\begin{aligned}
z^{\hat{\Delta} \breve{x}}(\breve{x}, \breve{y})= & \int_{0}^{\breve{y}} f(\breve{x}, \breve{t}) \bar{\varphi}(u(\breve{x}, \breve{t})) \hat{\Delta} \breve{t} \\
& +\int_{0}^{\breve{y}} b(\breve{x}, \breve{t})\left(h(\breve{x}, \breve{t}) \bar{\varphi}(u(\breve{x}, \breve{t}))+\int_{0}^{\breve{x}} g(\breve{\tau}, \breve{t}) \bar{\varphi}(u(\breve{\tau}, \breve{t})) \hat{\Delta} \breve{\tau}\right) \hat{\Delta} \breve{t} \\
\leq & \int_{0}^{\breve{y}} f(\breve{x}, \breve{t}) \bar{\varphi} \circ \bar{\psi}^{-1}(z(\breve{x}, \breve{t})) \hat{\Delta} \breve{t}+\int_{0}^{\check{y}} b(\breve{x}, \breve{t}) \\
& \times\left(h(\breve{x}, \breve{t}) \bar{\varphi} \circ \bar{\psi}^{-1}(z(\breve{x}, \breve{t}))+\int_{0}^{\breve{x}} g(\breve{\tau}, \breve{t}) \bar{\varphi} \circ \bar{\psi}^{-1}(z(\breve{\tau}, \breve{t})) \hat{\Delta} \breve{\tau}\right) \hat{\Delta} \breve{t} \\
\leq & \bar{\varphi} \circ \bar{\psi}^{-1}(z(\breve{x}, \breve{y}))\left[\int_{0}^{\breve{y}} f(\breve{x}, \breve{t}) \hat{\Delta} \breve{t}\right. \\
& \left.+\int_{0}^{\breve{y}} b(\breve{x}, \breve{t})\left(h(\breve{x}, \breve{t})+\int_{0}^{\breve{x}} g(\breve{\tau}, \breve{t}) \hat{\Delta} \breve{\tau}\right)\right] \hat{\Delta} \breve{t},
\end{aligned}
$$

then

$$
\frac{z^{\hat{\Delta} \breve{x}}(\breve{x}, \breve{y})}{\bar{\varphi} \circ \bar{\psi}^{-1}(z(\breve{x}, \breve{y}))} \leq\left[\int_{0}^{\breve{y}} f(\breve{x}, \breve{t}) \hat{\Delta} \breve{t}+\int_{0}^{\check{y}} b(\breve{x}, \breve{t})\left(h(\breve{x}, \breve{t})+\int_{0}^{\breve{x}} g(\breve{\tau}, \breve{t}) \hat{\Delta} \breve{\tau}\right)\right] \hat{\Delta} \breve{t} .
$$

Integrating (2.12) and using (2.3) and (2.11), we get

$$
\breve{G}(z(\breve{x}, \breve{y})) \leq \breve{G}(q(\breve{\xi}, \breve{\zeta}))+\breve{A}(\breve{x}, \breve{y})+\int_{0}^{\breve{x}} \int_{0}^{\breve{y}} f(\breve{s}, \breve{t}) \hat{\Delta} \breve{t} \hat{\Delta} \breve{s} .
$$

Since $(\breve{\xi}, \breve{\zeta}) \in \Omega$ is chosen arbitrarily,

$$
z(\breve{x}, \breve{y}) \leq \breve{G}^{-1}\left[\breve{G}(q(\breve{x}, \breve{y}))+\breve{A}(\breve{x}, \breve{y})+\int_{0}^{\breve{x}} \int_{0}^{\breve{y}} f(\breve{s}, \breve{t}) \hat{\Delta} \breve{t} \hat{\Delta} \breve{s}\right]
$$

From (2.13) and $u(\breve{x}, \breve{y}) \leq \bar{\psi}^{-1}(z(\breve{x}, \breve{y}))$, we get the required inequality in (2.10). For $a(\breve{x}, \breve{y})=$ 0 , we carry out the above procedure with $\epsilon>0$ instead of $a(\breve{x}, \breve{y})$ and subsequently let $\epsilon \rightarrow 0$. This completes the proof. 
Corollary 2.5 If we take $\breve{\mathbb{T}}=\mathbb{R}$ in Theorem 2.4 , then the inequality

$$
\begin{aligned}
\bar{\psi}(u(\breve{x}, \breve{y})) \leq & a(\breve{x}, \breve{y})+\int_{0}^{\breve{x}} \int_{0}^{\breve{y}}[f(\breve{s}, \breve{t}) \bar{\varphi}(u(\breve{s}, \breve{t}))+p(\breve{s}, \breve{t})] d \breve{t} d \breve{s} \\
& +\int_{0}^{\breve{x}} \int_{0}^{\breve{y}} b(\breve{s}, \breve{t})\left[h(\breve{s}, \breve{t}) \bar{\varphi}(u(\breve{s}, \breve{t}))+\int_{0}^{\breve{s}} g(\breve{\tau}, \breve{t}) \bar{\varphi}(u(\breve{\tau}, \breve{t})) d \breve{\tau}\right] d \breve{t} d \breve{s},
\end{aligned}
$$

for $(\breve{x}, \breve{y}) \in \Omega$, implies

$$
u(\breve{x}, \breve{y}) \leq \bar{\psi}^{-1}\left\{\breve{G}^{-1}\left[\breve{G}(q(\breve{x}, \breve{y}))+A(\breve{x}, \breve{y})+\int_{0}^{\breve{x}} \int_{0}^{\breve{y}} f(\breve{s}, \breve{t}) d \breve{t} d \breve{s}\right]\right\}
$$

for $0 \leq \breve{x} \leq \breve{x}_{1}, 0 \leq \breve{y} \leq \breve{y}_{1}$, where $\breve{G}$ is defined by (2.8) and

$$
\breve{A}(\breve{x}, \breve{y})=\int_{0}^{\breve{x}} \int_{0}^{\breve{y}} b(\breve{s}, \breve{t})\left[h(\breve{s}, \breve{t})+\int_{0}^{\breve{s}} g(\breve{\tau}, \breve{t}) d \breve{\tau}\right] d \breve{t} d \breve{s}
$$

and $\left(\breve{x}_{1}, \breve{y}_{1}\right) \in \Omega$ is chosen so that

$$
\left(\breve{G}(q(\breve{x}, \breve{y}))+\breve{A}(\breve{x}, \breve{y})+\int_{0}^{\breve{x}} \int_{0}^{\breve{y}} f(\breve{s}, \breve{t}) d \breve{t} d \breve{s}\right) \in \operatorname{Dom}\left(\breve{G}^{-1}\right) .
$$

Corollary 2.6 The discrete form can be obtained by letting $\breve{\mathbb{T}}=\mathbb{Z}$ in Theorem 2.4:

$$
\begin{aligned}
\bar{\psi}(u(\breve{x}, \breve{y})) \leq & a(\breve{x}, \breve{y})+\sum_{\breve{s}=0}^{\breve{x}-1} \sum_{\breve{t}=0}^{\breve{y}-1}[f(\breve{s}, \breve{t}) \bar{\varphi}(u(\breve{s}, \breve{t}))+p(\breve{s}, \breve{t})] \\
& +\sum_{\breve{s}=0}^{\breve{x}-1} \sum_{\breve{t}=0}^{\breve{y}-1} b(\breve{s}, \breve{t})\left[h(\breve{s}, \breve{t}) \bar{\varphi}(u(\breve{s}, \breve{t}))+\sum_{\breve{t}=0}^{\breve{s}-1} g(\breve{\tau}, \breve{t}) \bar{\varphi}(u(\breve{\tau}, \breve{t}))\right],
\end{aligned}
$$

for $(\breve{x}, \breve{y}) \in \Omega$, implies

$$
u(\breve{x}, \breve{y}) \leq \bar{\psi}^{-1}\left\{G^{-1}\left[G(q(\breve{x}, \breve{y}))+A(\breve{x}, \breve{y})+\sum_{\breve{s}=0}^{\breve{x}-1} \sum_{\breve{t}=0}^{\breve{y}-1} f(\breve{s}, \breve{t})\right]\right\}
$$

for $0 \leq \breve{x} \leq \breve{x}_{1}, 0 \leq \breve{y} \leq \breve{y}_{1}$, where $\breve{G}$ is defined by (2.9) and

$$
\breve{A}(\breve{x}, \breve{y})=\sum_{\breve{s}=0}^{\breve{x}-1} \sum_{\breve{t}=0}^{\breve{y}-1} b(\breve{s}, \breve{t})\left[h(\breve{s}, \breve{t})+\sum_{\breve{\tau}=0}^{\breve{s}-1} g(\breve{\tau}, \breve{t})\right]
$$

and $\left(\breve{x}_{1}, \breve{y}_{1}\right) \in \Omega$ is chosen so that

$$
\left(\breve{G}(q(\breve{x}, \breve{y}))+\breve{A}(\breve{x}, \breve{y})+\sum_{\breve{s}=0}^{\breve{x}-1} \sum_{\breve{y}=0}^{\breve{y}-1} f(\breve{s}, \breve{t})\right) \in \operatorname{Dom}\left(\breve{G}^{-1}\right) .
$$


Theorem 2.7 Assume that $g, a, u, f, p, \bar{\psi}$ and $\bar{\varphi}$ are as in Theorem 2.1. If $u(\breve{x}, \breve{y})$ satisfies

$$
\begin{aligned}
\bar{\psi}(u(\breve{x}, \breve{y})) \leq & a(\breve{x}, \breve{y})+\int_{0}^{\breve{x}} \int_{0}^{\breve{y}} \bar{\varphi}(u(\breve{s}, \breve{t}))[f(\breve{s}, \breve{t}) \bar{\varphi}(u(\breve{s}, \breve{t}))+p(\breve{s}, \breve{t})] \hat{\Delta} \breve{t} \hat{\Delta} \breve{s} \\
& +\int_{0}^{\breve{x}} \int_{0}^{\breve{y}} f(\breve{s}, \breve{t}) \bar{\varphi}(u(\breve{s}, \breve{t}))\left(\int_{0}^{\breve{s}} g(\breve{\tau}, \breve{t}) \bar{\varphi}(u(\breve{\tau}, \breve{t})) \hat{\Delta} \breve{\tau}\right) \hat{\Delta} \breve{t} \hat{\Delta} \breve{s},
\end{aligned}
$$

for $(\breve{x}, \breve{y}) \in \Omega$, then

$$
\begin{aligned}
u(\breve{x}, \breve{y}) \leq & \bar{\psi}^{-1}\left\{\breve { G } ^ { - 1 } \left(\breve { F } ^ { - 1 } \left[\breve{F}\left(q_{1}(\breve{x}, \breve{y})\right)\right.\right.\right. \\
& \left.\left.\left.+\int_{0}^{\breve{x}} \int_{0}^{\breve{y}} f(\breve{s}, \breve{t})\left(1+\int_{0}^{\breve{s}} g(\breve{\tau}, \breve{t}) \hat{\Delta} \breve{\tau}\right) \hat{\Delta} \breve{t} \hat{\Delta} \breve{s}\right]\right)\right\},
\end{aligned}
$$

for $0 \leq \breve{x} \leq \breve{x}_{1}, 0 \leq \breve{y} \leq \breve{y}_{1}$, where $\breve{G}$ is defined in (2.3) and

$$
\begin{aligned}
& q_{1}(\breve{x}, \breve{y})=\breve{G}(a(\breve{x}, \breve{y}))+\int_{0}^{\breve{x}} \int_{0}^{\breve{y}} p(\breve{s}, \breve{t}) \hat{\Delta} \breve{t} \hat{\Delta} \breve{s}, \\
& \breve{F}(r)=\int_{r_{0}}^{r} \frac{\hat{\Delta} \breve{s}}{\left(\left(\bar{\varphi} \circ \bar{\psi}^{-1}\right) \circ \breve{G}^{-1}\right)(\breve{s})}, \quad r \geq r_{0}>0, \\
& \breve{F}(+\infty)=\int_{r_{0}}^{+\infty} \frac{\hat{\Delta} \breve{s}}{\left(\bar{\varphi} \circ \bar{\psi}^{-1}\right) \circ \breve{G}^{-1}(\breve{s})}=+\infty,
\end{aligned}
$$

and $\left(\breve{x}_{1}, \breve{y}_{1}\right) \in \Omega$ is chosen so that

$$
\left(\breve{F}\left(q_{1}(\breve{x}, \breve{y})\right)+\int_{0}^{\breve{x}} \int_{0}^{\breve{y}} f(\breve{s}, \breve{t})\left(1+\int_{0}^{\breve{s}} g(\breve{\tau}, \breve{t}) \hat{\Delta} \breve{\tau}\right) \hat{\Delta} \breve{t} \hat{\Delta} \breve{s}\right) \in \operatorname{Dom}\left(\breve{F}^{-1}\right) .
$$

Proof Suppose that $a(\breve{\xi}, \breve{\zeta})>0$. Fixing an arbitrary $(\breve{\xi}, \breve{\zeta}) \in \Omega$, we define a positive and nondecreasing function $z(\breve{x}, \breve{y})$ by

$$
\begin{aligned}
z(\breve{x}, \breve{y})= & a(\breve{\xi}, \breve{\zeta})+\int_{0}^{\breve{x}} \int_{0}^{\breve{y}} \bar{\varphi}(u(\breve{s}, \breve{t}))[f(\breve{s}, \breve{t}) \bar{\varphi}(u(\breve{s}, \breve{t}))+p(\breve{s}, \breve{t})] \hat{\Delta} \breve{t} \hat{\Delta} \breve{s} \\
& +\int_{0}^{\breve{x}} \int_{0}^{\breve{y}} f(\breve{s}, \breve{t}) \bar{\varphi}(u(\breve{s}, \breve{t}))\left(\int_{0}^{\breve{s}} g(\breve{\tau}, \breve{t}) \bar{\varphi}(u(\breve{\tau}, \breve{t})) \hat{\Delta} \breve{\tau}\right) \hat{\Delta} \breve{t} \hat{\Delta} \breve{s},
\end{aligned}
$$

for $0 \leq \breve{x} \leq \breve{\xi} \leq \breve{x}_{1}, 0 \leq \breve{y} \leq \breve{\zeta} \leq \breve{y}_{1}$, then $z(0, \breve{y})=z(\breve{x}, 0)=a(\breve{\xi}, \breve{\zeta})$ and

$$
u(\breve{x}, \breve{y}) \leq \bar{\psi}^{-1}(z(\breve{x}, \breve{y}))
$$

then we have

$$
\begin{aligned}
z^{\hat{\Delta} \breve{x}}(\breve{x}, \breve{y})= & \int_{0}^{\breve{y}} \bar{\varphi}(u(\breve{x}, \breve{t}))[f(\breve{x}, \breve{t}) \bar{\varphi}(u(\breve{x}, \breve{t}))+p(\breve{x}, \breve{t})] \hat{\Delta} \breve{t} \\
& +\int_{0}^{\breve{y}} f(\breve{x}, \breve{t}) \bar{\varphi}(u(\breve{x}, \breve{t}))\left(\int_{0}^{\breve{x}} g(\breve{\tau}, \breve{t}) \bar{\varphi}(u(\breve{\tau}, \breve{t})) \hat{\Delta} \breve{\tau}\right) \hat{\Delta} \breve{t}
\end{aligned}
$$




$$
\begin{aligned}
\leq & \int_{0}^{\breve{y}} \bar{\varphi} \circ \bar{\psi}^{-1}(z(\breve{x}, \breve{t}))\left[f(\breve{x}, \breve{t}) \bar{\varphi} \circ \bar{\psi}^{-1}(z(\breve{x}, \breve{t}))+p(\breve{x}, \breve{t})\right] \hat{\Delta} \breve{t} \\
& +\int_{0}^{\breve{y}} f(\breve{x}, \breve{t}) \bar{\varphi} \circ \bar{\psi}^{-1}(z(\breve{x}, \breve{t}))\left(\int_{0}^{\breve{x}} g(\breve{\tau}, \breve{t}) \bar{\varphi} \circ \bar{\psi}^{-1}(z(\breve{\tau}, \breve{t})) \hat{\Delta} \breve{\tau}\right) \hat{\Delta} \breve{t} \\
\leq & \bar{\varphi} \circ \bar{\psi}^{-1}(z(\breve{x}, \breve{y})) \int_{0}^{\check{y}}\left[f(\breve{x}, \breve{t}) \bar{\varphi} \circ \bar{\psi}^{-1}(z(\breve{x}, \breve{t}))+p(\breve{x}, \breve{t})\right] \hat{\Delta} \breve{t} \\
& +\bar{\varphi} \circ \bar{\psi}^{-1}(z(\breve{x}, \breve{y})) \int_{0}^{\check{y}} f(\breve{x}, \breve{t})\left(\int_{0}^{\breve{x}} g(\breve{\tau}, \breve{t}) \bar{\varphi} \circ \bar{\psi}^{-1}(z(\breve{\tau}, \breve{t})) \hat{\Delta} \breve{\tau}\right) \hat{\Delta} \breve{t},
\end{aligned}
$$

or

$$
\begin{aligned}
\frac{z^{\hat{\Delta} \breve{x}(\breve{x}, \breve{y})}}{\bar{\varphi} \circ \bar{\psi}^{-1}(z(\breve{x}, \breve{y}))} \leq & \int_{0}^{\check{y}}\left[f(\breve{x}, \breve{t}) \bar{\varphi} \circ \bar{\psi}^{-1}(z(\breve{x}, \breve{t}))+p(\breve{x}, \breve{t})\right] \hat{\Delta} \breve{t} \\
& +\int_{0}^{\check{y}} f(\breve{x}, \breve{t})\left(\int_{0}^{\breve{x}} g(\breve{t}, \breve{t}) \bar{\varphi} \circ \bar{\psi}^{-1}(z(\breve{\tau}, \breve{t})) \hat{\Delta} \breve{t}\right) \hat{\Delta} \breve{t} .
\end{aligned}
$$

Integrating (2.17) and using (2.3), we get

$$
\begin{aligned}
\breve{G}(z(\breve{x}, \breve{y})) \leq & \breve{G}(a(\breve{\xi}, \breve{\zeta}))+\int_{0}^{\breve{x}} \int_{0}^{\breve{y}}\left[f(\breve{s}, \breve{t}) \bar{\varphi} \circ \bar{\psi}^{-1}(z(\breve{s}, \breve{t}))+p(\breve{s}, \breve{t})\right] \hat{\Delta} \breve{t} \hat{\Delta} \breve{s} \\
& +\int_{0}^{\breve{x}} \int_{0}^{\breve{y}} f(\breve{s}, \breve{t})\left(\int_{0}^{\breve{s}} g(\breve{\tau}, \breve{t}) \bar{\varphi} \circ \bar{\psi}^{-1}(z(\breve{\tau}, \breve{t})) \hat{\Delta} \breve{\tau}\right) \hat{\Delta} \breve{t} \hat{\Delta} \breve{s} .
\end{aligned}
$$

$(\breve{\xi}, \breve{\zeta}) \in \Omega$ is chosen arbitrarily, then from (2.15) we have

$$
\begin{aligned}
\breve{G}(z(\breve{x}, \breve{y})) \leq & q_{1}(\breve{x}, \breve{y})+\int_{0}^{\breve{x}} \int_{0}^{\breve{y}} f(\breve{s}, \breve{t}) \bar{\varphi} \circ \bar{\psi}^{-1}(z(\breve{s}, \breve{t})) \hat{\Delta} \breve{t} \hat{\Delta} \breve{s} \\
& +\int_{0}^{\breve{x}} \int_{0}^{\breve{y}} f(\breve{s}, \breve{t})\left(\int_{0}^{\breve{s}} g(\breve{\tau}, \breve{t}) \bar{\varphi} \circ \bar{\psi}^{-1}(z(\breve{\tau}, \breve{t})) \hat{\Delta} \breve{\tau}\right) \hat{\Delta} \breve{t} \hat{\Delta} \breve{s}
\end{aligned}
$$

Since $q_{1}(\breve{x}, \breve{y})>0$ is a nondecreasing function, fixing an arbitrary point $(\breve{\xi}, \breve{\zeta}) \in \Omega$ and defining $v(\breve{x}, \breve{y})>0$ to be a nondecreasing function by

$$
\begin{aligned}
v(\breve{x}, \breve{y})= & q_{1}(\breve{\xi}, \breve{\zeta})+\int_{0}^{\breve{x}} \int_{0}^{\breve{y}} f(\breve{s}, \breve{t}) \bar{\varphi} \circ \bar{\psi}^{-1}(z(\breve{s}, \breve{t})) \hat{\Delta} \breve{t} \hat{\Delta} \breve{s} \\
& +\int_{0}^{\breve{x}} \int_{0}^{\breve{y}} f(\breve{s}, \breve{t})\left(\int_{0}^{\breve{s}} g(\breve{\tau}, \breve{t}) \bar{\varphi} \circ \bar{\psi}^{-1}(z(\breve{\tau}, \breve{t})) \hat{\Delta} \breve{\tau}\right) \hat{\Delta} \breve{t} \hat{\Delta} \breve{s},
\end{aligned}
$$

for $0 \leq \breve{x} \leq \breve{\xi} \leq \breve{x}_{1}, 0 \leq \breve{y} \leq \breve{\zeta} \leq y_{1}$, we have $v(0, \breve{y})=v(\breve{x}, 0)=q_{1}(\breve{\xi}, \breve{\zeta})$ and

$$
z(\breve{x}, \breve{y}) \leq \breve{G}^{-1}(v(\breve{x}, \breve{y}))
$$

then we have

$$
\begin{aligned}
v^{\hat{\Delta} \breve{x}}(\breve{x}, \breve{y})= & \int_{0}^{\breve{y}} f(\breve{x}, \breve{t}) \bar{\varphi} \circ \bar{\psi}^{-1}(z(\breve{x}, \breve{t})) \hat{\Delta} \breve{t} \\
& +\int_{0}^{\breve{y}} f(\breve{x}, \breve{t})\left(\int_{0}^{\breve{x}} g(\breve{\tau}, \breve{t}) \bar{\varphi} \circ \bar{\psi}^{-1}(z(\breve{t}, \breve{t})) \hat{\Delta} \breve{t}\right) \hat{\Delta} \breve{t}
\end{aligned}
$$




$$
\begin{aligned}
\leq & \int_{0}^{\breve{y}} f(\breve{x}, \breve{t}) \bar{\varphi} \circ \bar{\psi}^{-1}\left(G^{-1}(v(\breve{x}, \breve{t}))\right) \hat{\Delta} \breve{t} \\
& +\int_{0}^{\breve{y}} f(\breve{x}, \breve{t})\left(\int_{0}^{\check{x}} g(\breve{\tau}, \breve{t}) \bar{\varphi} \circ \bar{\psi}^{-1}\left(G^{-1}(v(\breve{\tau}, \breve{t}))\right) \hat{\Delta} \breve{\tau}\right) \hat{\Delta} \breve{t} \\
\leq & \left(\bar{\varphi} \circ \bar{\psi}^{-1}\right) \circ \breve{G}^{-1}(v(\breve{x}, \breve{y}))\left[\int_{0}^{\breve{y}} f(\breve{x}, \breve{t}) \hat{\Delta} \breve{t}+\int_{0}^{\breve{y}} f(\breve{x}, \breve{t})\left(\int_{0}^{\breve{x}} g(\breve{\tau}, \breve{t}) \hat{\Delta} \breve{\tau}\right) \hat{\Delta} \breve{t}\right],
\end{aligned}
$$

or

$$
\frac{v^{\hat{\Delta} \breve{x}}(\breve{x}, \breve{y})}{\left(\bar{\varphi} \circ \bar{\psi}^{-1}\right) \circ \breve{G}^{-1}(v(\breve{x}, \breve{y}))} \leq\left[\int_{0}^{\breve{y}} f(\breve{x}, \breve{t}) \hat{\Delta} \breve{t}+\int_{0}^{\breve{y}} f(\breve{x}, \breve{t})\left(\int_{0}^{\breve{x}} g(\breve{\tau}, \breve{t}) \hat{\Delta} \breve{t}\right) \hat{\Delta} \breve{t}\right] .
$$

Integrating (2.19) and using (2.16), we get

$$
\breve{F}(v(\breve{x}, \breve{y})) \leq \breve{F}\left(q_{1}(\breve{\xi}, \breve{\zeta})\right)+\int_{0}^{\breve{x}} \int_{0}^{\breve{y}} f(\breve{s}, \breve{t})\left[1+\int_{0}^{\breve{s}} g(\breve{\tau}, \breve{t}) \hat{\Delta} \breve{\tau}\right] \hat{\Delta} \breve{t} \hat{\Delta} \breve{s} .
$$

Since we can choose $(\breve{\xi}, \breve{\zeta}) \in \Omega$ arbitrarily, we have

$$
v(\breve{x}, \breve{y}) \leq \breve{F}^{-1}\left[\breve{F}\left(q_{1}(\breve{x}, \breve{y})\right)+\int_{0}^{\breve{x}} \int_{0}^{\breve{y}} f(\breve{s}, \breve{t})\left[1+\int_{0}^{\breve{s}} g(\breve{\tau}, \breve{t}) \hat{\Delta} \breve{\tau}\right] \hat{\Delta} \breve{t} \hat{\Delta} \breve{s}\right] .
$$

From (2.20), (2.18) and $u(\breve{x}, \breve{y}) \leq \bar{\psi}^{-1}(z(\breve{x}, \breve{y}))$ we get the desired inequality in (2.14). For $a(\breve{x}, \breve{y})=0$, we carry out the above procedure with $\epsilon>0$ instead of $a(\breve{x}, \breve{y})$ and subsequently let $\epsilon \rightarrow 0$. This completes the proof.

Corollary 2.8 If we take $\breve{\mathbb{T}}=\mathbb{R}$ in Theorem 2.7 , then the inequality

$$
\begin{aligned}
\bar{\psi}(u(\breve{x}, \breve{y})) \leq & a(\breve{x}, \breve{y})+\int_{0}^{\breve{x}} \int_{0}^{\breve{y}} \bar{\varphi}(u(\breve{s}, \breve{t}))[f(\breve{s}, \breve{t}) \bar{\varphi}(u(\breve{s}, \breve{t}))+p(\breve{s}, \breve{t})] d \breve{t} d \breve{s} \\
& +\int_{0}^{\breve{x}} \int_{0}^{\breve{y}} f(\breve{s}, \breve{t}) \bar{\varphi}(u(\breve{s}, \breve{t}))\left(\int_{0}^{\breve{s}} g(\breve{\tau}, \breve{t}) \bar{\varphi}(u(\breve{\tau}, \breve{t})) \hat{\Delta} \breve{\tau}\right) d \breve{t} d \breve{s}
\end{aligned}
$$

for $(\breve{x}, \breve{y}) \in \Omega$, implies

$$
u(\breve{x}, \breve{y}) \leq \bar{\psi}^{-1}\left\{\breve{G}^{-1}\left(\breve{F}^{-1}\left[\breve{F}\left(q_{2}(\breve{x}, \breve{y})\right)+\int_{0}^{\breve{x}} \int_{0}^{\breve{y}} f(\breve{s}, \breve{t})\left(1+\int_{0}^{\breve{s}} g(\breve{\tau}, \breve{t}) d \breve{\tau}\right) d \breve{t} d \breve{s}\right]\right)\right\},
$$

for $0 \leq \breve{x} \leq \breve{x}_{1}, 0 \leq \breve{y} \leq \breve{y}_{1}$, where $\breve{G}$ is as defined in (2.8) and

$$
\begin{aligned}
& q_{2}(\breve{x}, \breve{y})=\breve{G}(a(\breve{x}, \breve{y}))+\int_{0}^{\breve{x}} \int_{0}^{\breve{y}} p(\breve{s}, \breve{t}) d \breve{t} d \breve{s}, \\
& \breve{F}(r)=\int_{r_{0}}^{r} \frac{d \breve{s}}{\left(\left(\bar{\varphi} \circ \bar{\psi}^{-1}\right) \circ \breve{G}^{-1}\right)(\breve{s})}, \quad r \geq r_{0}>0, \\
& \breve{F}(+\infty)=\int_{r_{0}}^{+\infty} \frac{d \breve{s}}{\left(\bar{\varphi} \circ \bar{\psi}^{-1}\right) \circ \breve{G}^{-1}(\breve{s})}=+\infty,
\end{aligned}
$$


and $\left(\breve{x}_{1}, \breve{y}_{1}\right) \in \Omega$ is chosen so that

$$
\left(\breve{F}\left(q_{2}(\breve{x}, \breve{y})\right)+\int_{0}^{\breve{x}} \int_{0}^{\breve{y}} f(\breve{s}, \breve{t})\left(1+\int_{0}^{\breve{s}} g(\breve{\tau}, \breve{t}) d \breve{\tau}\right) d \breve{t} d \breve{s}\right) \in \operatorname{Dom}\left(\breve{F}^{-1}\right) .
$$

Corollary 2.9 The discrete form of Theorem 2.7 can be obtained by letting $\breve{\mathbb{T}}=\mathbb{Z}$ :

$$
\begin{aligned}
\bar{\psi}(u(\breve{x}, \breve{y})) \leq & a(\breve{x}, \breve{y})+\sum_{\breve{s}=0}^{\breve{x}-1} \sum_{\breve{t}=0}^{\breve{y}-1} \bar{\varphi}(u(\breve{s}, \breve{t}))[f(\breve{s}, \breve{t}) \bar{\varphi}(u(\breve{s}, \breve{t}))+p(\breve{s}, \breve{t})] \\
& +\sum_{\breve{s}=0}^{\breve{x}-1} \sum_{\breve{t}=0}^{\breve{y}-1} f(\breve{s}, \breve{t}) \bar{\varphi}(u(\breve{s}, \breve{t}))\left(\sum_{\breve{\tau}=0}^{\breve{s}-1} g(\breve{t}, \breve{t}) \bar{\varphi}(u(\breve{\tau}, \breve{t}))\right)
\end{aligned}
$$

for $(\breve{x}, \breve{y}) \in \Omega$, implies

$$
u(\breve{x}, \breve{y}) \leq \bar{\psi}^{-1}\left\{\bar{G}^{-1}\left(\bar{F}^{-1}\left[\bar{F}\left(\bar{q}_{2}(\breve{x}, \breve{y})\right)+\sum_{\breve{s}=0}^{\breve{x}-1} \sum_{\breve{t}=0}^{\breve{y}-1} f(\breve{s}, \breve{t})\left(1+\sum_{\breve{\tau}=0}^{\breve{s}-1} g(\breve{\tau}, \breve{t})\right)\right]\right)\right\},
$$

for $0 \leq \breve{x} \leq \breve{x}_{1}, 0 \leq \breve{y} \leq \breve{y}_{1}$, where $\breve{G}$ is as defined in (2.9) and

$$
\begin{aligned}
& \bar{q}_{2}(\breve{x}, \breve{y})=\breve{G}(a(\breve{x}, \breve{y}))+\sum_{\breve{s}=0}^{\breve{x}-1} \sum_{\breve{t}=0}^{\breve{y}-1} p(\breve{s}, \breve{t}), \\
& \bar{F}(r)=\sum_{\breve{s}=r_{0}}^{r-1} \frac{1}{\left(\left(\bar{\varphi} \circ \bar{\psi}^{-1}\right) \circ \bar{G}^{-1}\right)(\breve{s})}, \quad r \geq r_{0}>0, \\
& \bar{F}(+\infty)=\sum_{\breve{s}=r_{0}}^{+\infty} \frac{1}{\left(\bar{\varphi} \circ \bar{\psi}^{-1}\right) \circ \bar{G}^{-1}(\breve{s})}=+\infty,
\end{aligned}
$$

and $\left(\breve{x}_{1}, \breve{y}_{1}\right) \in \Omega$ is chosen so that

$$
\left(\bar{F}\left(\bar{q}_{2}(\breve{x}, \breve{y})\right)+\sum_{\breve{s}=0}^{\breve{x}-1} \sum_{\breve{t}=0}^{\breve{y}-1} f(\breve{s}, \breve{t})\left(1+\sum_{\breve{\tau}=0}^{\breve{s}-1} g(\breve{\tau}, \breve{t})\right)\right) \in \operatorname{Dom}\left(\bar{F}^{-1}\right) .
$$

Theorem 2.10 Assume that $g, a, f, u, \bar{\psi}$ and $\bar{\varphi}$ be as in Theorem 2.1. If $u(\breve{x}, \breve{y})$ satisfies

$$
\begin{aligned}
\bar{\psi}(u(\breve{x}, \breve{y})) \leq & a(\breve{x}, \breve{y})+\left(\int_{0}^{\breve{x}} \int_{0}^{\breve{y}} f(\breve{s}, \breve{t}) \bar{\varphi}(u(\breve{s}, \breve{t})) \hat{\Delta} \breve{t} \hat{\Delta} \breve{s}\right)^{2} \\
& +\int_{0}^{\breve{x}} \int_{0}^{\breve{y}} f(\breve{s}, \breve{t}) \bar{\varphi}(u(\breve{s}, \breve{t}))\left(\int_{0}^{\breve{s}} g(\breve{\tau}, \breve{t}) \bar{\varphi}(u(\breve{\tau}, \breve{t})) \hat{\Delta} \breve{\tau}\right) \hat{\Delta} \breve{t} \hat{\Delta} \breve{s},
\end{aligned}
$$

for $(\breve{x}, \breve{y}) \in \Omega$, then

$$
u(\breve{x}, \breve{y}) \leq \bar{\psi}^{-1}\left\{\breve{H}^{-1}\left[\breve{H}(a(\breve{x}, \breve{y}))+\breve{B}(\breve{x}, \breve{y})+\left(\int_{0}^{\breve{x}} \int_{0}^{\breve{y}} f(\breve{s}, \breve{t}) \hat{\Delta} \breve{t} \hat{\Delta} \breve{s}\right)^{2}\right]\right\},
$$


for $0 \leq \breve{x} \leq \breve{x}_{1}, 0 \leq \breve{y} \leq \breve{y}_{1}$, where

$$
\begin{aligned}
& \breve{B}(\breve{x}, \breve{y})=\int_{0}^{\breve{x}} \int_{0}^{\breve{y}} f(\breve{s}, \breve{t})\left(\int_{0}^{\breve{s}} g(\breve{\tau}, \breve{t}) \hat{\Delta} \breve{\tau}\right) \hat{\Delta} \breve{t} \hat{\Delta} \breve{s}, \\
& \breve{H}(r)=\int_{r_{0}}^{r} \frac{\hat{\Delta} \breve{s}}{\left(\bar{\varphi} \circ \bar{\psi}^{-1}\right)^{2}(\breve{s})}, \quad r \geq r_{0}>0 \\
& \breve{H}(+\infty)=\int_{r_{0}}^{+\infty} \frac{\hat{\Delta} \breve{s}}{\left(\bar{\varphi} \circ \bar{\psi}^{-1}\right)^{2}(\breve{s})}=+\infty
\end{aligned}
$$

and $\left(\breve{x}_{1}, \breve{y}_{1}\right) \in \Omega$ is chosen so that

$$
\left(\breve{H}(a(\breve{x}, \breve{y}))+B(\breve{x}, \breve{y})+\left(\int_{0}^{\breve{x}} \int_{0}^{\breve{y}} f(\breve{s}, \breve{t}) \hat{\Delta} \breve{t} \hat{\Delta} \breve{s}\right)^{2}\right) \in \operatorname{Dom}\left(\breve{H}^{-1}\right) .
$$

Proof Assume that $a(\breve{x}, \breve{y})>0$. Taking $(\breve{\xi}, \breve{\zeta}) \in \Omega$ as a fixed arbitrary point, we define $z(\breve{x}, \breve{y})>0$ to be a nondecreasing function by

$$
\begin{aligned}
z(\breve{x}, \breve{y})= & a(\breve{\xi}, \breve{\zeta})+\left(\int_{0}^{\breve{x}} \int_{0}^{\breve{y}} f(\breve{s}, \breve{t}) \bar{\varphi}(u(\breve{s}, \breve{t})) \hat{\Delta} \breve{t} \hat{\Delta} \breve{s}\right)^{2} \\
& +\int_{0}^{\breve{x}} \int_{0}^{\breve{y}} f(\breve{s}, \breve{t}) \bar{\varphi}(u(\breve{s}, \breve{t}))\left(\int_{0}^{\breve{s}} g(\breve{\tau}, \breve{t}) \bar{\varphi}(u(\breve{\tau}, \breve{t})) \hat{\Delta} \breve{\tau}\right) \hat{\Delta} \breve{t} \hat{\Delta} \breve{s},
\end{aligned}
$$

for $0 \leq \breve{x} \leq \breve{\xi} \leq \breve{x}_{1}, 0 \leq \breve{y} \leq \breve{\zeta} \leq \breve{y}_{1}$, hence $z(0, \breve{y})=z(\breve{x}, 0)=a(\breve{\xi}, \breve{\zeta})$ and

$$
u(\breve{x}, \breve{y}) \leq \bar{\psi}^{-1}(z(\breve{x}, \breve{y}))
$$

From (2.24), and applying the chain rule on time scales, Theorem 1.4, we get

$$
\begin{aligned}
& z^{\hat{\Delta} \breve{x}}(\breve{x}, \breve{y})=2\left(\int_{0}^{c} \int_{0}^{\breve{y}} f(\breve{s}, \breve{t}) \bar{\varphi}(u(\breve{s}, \breve{t})) \hat{\Delta} \breve{t} \hat{\Delta} \breve{s}\right) \int_{0}^{\breve{y}} f(\breve{x}, \breve{t}) \bar{\varphi}(u(\breve{x}, \breve{t})) \hat{\Delta} \breve{t} \\
& +\int_{0}^{\breve{y}} f(\breve{x}, \breve{t}) \bar{\varphi}(u(\breve{x}, \breve{t}))\left(\int_{0}^{\breve{x}} g(\breve{\tau}, \breve{t}) \bar{\varphi}(u(\breve{\tau}, \breve{t})) \hat{\Delta} \breve{\tau}\right) \hat{\Delta} \breve{t} \\
& \leq 2\left(\int_{0}^{c} \int_{0}^{\breve{y}} f(\breve{s}, \breve{t}) \bar{\varphi} \circ \bar{\psi}^{-1}(z(\breve{s}, \breve{t})) \hat{\Delta} \breve{t} \hat{\Delta} \breve{s}\right) \int_{0}^{\breve{y}} f(\breve{x}, \breve{t}) \bar{\varphi} \circ \bar{\psi}^{-1}(z(\breve{x}, \breve{t})) \hat{\Delta} \breve{t} \\
& +\int_{0}^{\breve{y}} f(\breve{x}, \breve{t}) \bar{\varphi} \circ \bar{\psi}^{-1}(z(\breve{x}, \breve{t}))\left(\int_{0}^{\breve{x}} g(\breve{\tau}, \breve{t}) \bar{\varphi} \circ \bar{\psi}^{-1}(z(\breve{\tau}, \breve{t})) \hat{\Delta} \breve{\tau}\right) \hat{\Delta} \breve{t} \\
& \leq 2\left(\bar{\varphi} \circ \bar{\psi}^{-1}(z(\breve{x}, \breve{y}))\right)^{2}\left(\int_{0}^{c} \int_{0}^{\breve{y}} f(\breve{s}, \breve{t}) \hat{\Delta} \breve{t} \hat{\Delta} \breve{s}\right) \int_{0}^{\breve{y}} f(\breve{x}, \breve{t}) \hat{\Delta} \breve{t} \\
& +\left(\bar{\varphi} \circ \bar{\psi}^{-1}(z(\breve{x}, \breve{y}))\right)^{2} \int_{0}^{\breve{y}} f(\breve{x}, \breve{t})\left(\int_{0}^{\breve{x}} g(\breve{\tau}, \breve{t}) \hat{\Delta} \breve{\tau}\right) \hat{\Delta} \breve{t},
\end{aligned}
$$

thus, we have

$$
\begin{aligned}
\frac{z^{\hat{\Delta} \breve{x}}(\breve{x}, \breve{y})}{\left(\bar{\varphi} \circ \bar{\psi}^{-1}(z(\breve{x}, \breve{y}))\right)^{2}} \leq & 2\left(\int_{0}^{c} \int_{0}^{\breve{y}} f(\breve{s}, \breve{t}) \hat{\Delta} \breve{t} \hat{\Delta} \breve{s}\right) \int_{0}^{\breve{y}} f(\breve{x}, \breve{t}) \hat{\Delta} \breve{t} \\
& +\int_{0}^{\breve{y}} f(\breve{x}, \breve{t})\left(\int_{0}^{\breve{x}} g(\breve{\tau}, \breve{t}) \hat{\Delta} \breve{t}\right) \hat{\Delta} \breve{t}
\end{aligned}
$$




$$
\begin{aligned}
= & {\left[\left(\int_{0}^{\breve{x}} \int_{0}^{\breve{y}} f(\breve{s}, \breve{t}) \hat{\Delta} \breve{t} \hat{\Delta} \breve{s}\right)^{2}\right]^{\hat{\Delta}_{\breve{x}}} } \\
& +\int_{0}^{\breve{y}} f(\breve{x}, \breve{t})\left(\int_{0}^{\breve{x}} g(\breve{\tau}, \breve{t}) \hat{\Delta} \breve{t}\right) \hat{\Delta} \breve{t} .
\end{aligned}
$$

Integrating (2.25) and using (2.23), we get

$$
\begin{aligned}
\breve{H}(z(\breve{x}, \breve{y})) \leq & \breve{H}(a(\breve{\xi}, \breve{\zeta}))+\left(\int_{0}^{\breve{x}} \int_{0}^{\breve{y}} f(\breve{s}, \breve{t}) \hat{\Delta} \breve{t} \hat{\Delta} \breve{s}\right)^{2} \\
& +\int_{0}^{\breve{x}} \int_{0}^{\breve{y}} f(\breve{s}, \breve{t})\left(\int_{0}^{\breve{s}} g(\breve{\tau}, \breve{t}) \hat{\Delta} \breve{\tau}\right) \hat{\Delta} \breve{t} \hat{\Delta} \breve{s} .
\end{aligned}
$$

Since $(\breve{\xi}, \breve{\zeta}) \in \Omega$ is chosen arbitrarily,

$$
z(\breve{x}, \breve{y}) \leq \breve{H}^{-1}\left[\breve{H}(a(\breve{x}, \breve{y}))+\breve{B}(\breve{x}, \breve{y})+\left(\int_{0}^{\breve{x}} \int_{0}^{\breve{y}} f(\breve{s}, \breve{t}) \hat{\Delta} \breve{t} \hat{\Delta} \breve{s}\right)^{2}\right]
$$

From (2.26) and $u(\breve{x}, \breve{y}) \leq \bar{\psi}^{-1}(z(\breve{x}, \breve{y}))$, we get the desired inequality (2.21). For $a(\breve{x}, \breve{y})=0$, we carry out the above procedure with $\epsilon>0$ instead of $a(\breve{x}, \breve{y})$ and subsequently let $\epsilon \rightarrow 0$. This completes the proof.

Corollary 2.11 If we take $\breve{\mathbb{T}}=\mathbb{R}$ in Theorem 2.10 , then the inequality

$$
\begin{aligned}
\bar{\psi}(u(\breve{x}, \breve{y})) \leq & a(\breve{x}, \breve{y})+\left(\int_{0}^{\breve{x}} \int_{0}^{\breve{y}} f(\breve{s}, \breve{t}) \bar{\varphi}(u(\breve{s}, \breve{t})) d \breve{t} d \breve{s}\right)^{2} \\
& +\int_{0}^{\breve{x}} \int_{0}^{\breve{y}} f(\breve{s}, \breve{t}) \bar{\varphi}(u(\breve{s}, \breve{t}))\left(\int_{0}^{\breve{s}} g(\breve{\tau}, \breve{t}) \bar{\varphi}(u(\breve{\tau}, \breve{t})) d \breve{\tau}\right) d \breve{t} d \breve{s},
\end{aligned}
$$

for $(\breve{x}, \breve{y}) \in \Omega$, implies

$$
u(\breve{x}, \breve{y}) \leq \bar{\psi}^{-1}\left\{\breve{H}^{-1}\left[\breve{H}(a(\breve{x}, \breve{y}))+\breve{B}(\breve{x}, \breve{y})+\left(\int_{0}^{\breve{x}} \int_{0}^{\breve{y}} f(\breve{s}, \breve{t}) d \breve{t} d \breve{s}\right)^{2}\right]\right\},
$$

for $0 \leq \breve{x} \leq \breve{x}_{1}, 0 \leq \breve{y} \leq \breve{y}_{1}$, where

$$
\begin{aligned}
& \breve{B}(\breve{x}, \breve{y})=\int_{0}^{\breve{x}} \int_{0}^{\breve{y}} f(\breve{s}, \breve{t})\left(\int_{0}^{\breve{s}} g(\breve{\tau}, \breve{t}) d \breve{\tau}\right) d \breve{t} d \breve{s}, \\
& \breve{H}(r)=\int_{r_{0}}^{r} \frac{d \breve{s}}{\left(\bar{\varphi} \circ \bar{\psi}^{-1}\right)^{2}(\breve{s})}, \quad r \geq r_{0}>0, \quad \breve{H}(+\infty)=\int_{r_{0}}^{+\infty} \frac{d \breve{s}}{\left(\bar{\varphi} \circ \bar{\psi}^{-1}\right)^{2}(\breve{s})}=+\infty,
\end{aligned}
$$

and $\left(\breve{x}_{1}, \breve{y}_{1}\right) \in \Omega$ is chosen so that

$$
\left(\breve{H}(a(\breve{x}, \breve{y}))+B(\breve{x}, \breve{y})+\left(\int_{0}^{\breve{x}} \int_{0}^{\breve{y}} f(\breve{s}, \breve{t}) d \breve{t} d \breve{s}\right)^{2}\right) \in \operatorname{Dom}\left(\breve{H}^{-1}\right) .
$$


Corollary 2.12 The discrete form can be obtained by letting $\breve{\mathbb{T}}=\mathbb{Z}$ in Theorem 2.10 :

$$
\begin{aligned}
\bar{\psi}(u(\breve{x}, \breve{y})) \leq & a(\breve{x}, \breve{y})+\left(\sum_{\breve{s}=0}^{\breve{x}-1} \sum_{\breve{t}=0}^{\breve{y}-1} f(\breve{s}, \breve{t}) \bar{\varphi}(u(\breve{s}, \breve{t}))\right)^{2} \\
& +\sum_{\breve{s}=0}^{\breve{x}-1} \sum_{\breve{t}=0}^{\breve{y}-1} f(\breve{s}, \breve{t}) \bar{\varphi}(u(\breve{s}, \breve{t}))\left(\sum_{\breve{\tau}=0}^{\breve{s}-1} g(\breve{\tau}, \breve{t}) \bar{\varphi}(u(\breve{\tau}, \breve{t}))\right),
\end{aligned}
$$

for $(\breve{x}, \breve{y}) \in \Omega$, implies

$$
u(\breve{x}, \breve{y}) \leq \bar{\psi}^{-1}\left\{\breve{H}^{-1}\left[\breve{H}(a(\breve{x}, \breve{y}))+\breve{B}(\breve{x}, \breve{y})+\left(\sum_{\breve{s}=0}^{\breve{x}-1} \sum_{\breve{t}=0}^{\breve{y}-1} f(\breve{s}, \breve{t})\right)^{2}\right]\right\},
$$

for $0 \leq \breve{x} \leq \breve{x}_{1}, 0 \leq \breve{y} \leq \breve{y}_{1}$, where

$$
\begin{aligned}
& \breve{B}(\breve{x}, \breve{y})=\sum_{\breve{s}=0}^{\breve{x}-1} \sum_{\breve{t}=0}^{\breve{y}} f(\breve{s}, \breve{t})\left(\sum_{\breve{t}=0}^{\breve{s}-1} g(\breve{t}, \breve{t})\right), \\
& \breve{H}(r)=\sum_{\breve{s}=r_{0}}^{r-1} \frac{1}{\left(\bar{\varphi} \circ \bar{\psi}^{-1}\right)^{2}(\breve{s})}, \quad r \geq r_{0}>0, \quad \breve{H}(+\infty)=\sum_{\breve{s}=r_{0}}^{+\infty} \frac{1}{\left(\bar{\varphi} \circ \bar{\psi}^{-1}\right)^{2}(\breve{s})}=+\infty,
\end{aligned}
$$

and $\left(\breve{x}_{1}, \breve{y}_{1}\right) \in \Omega$ is chosen so that

$$
\left(\breve{H}(a(\breve{x}, \breve{y}))+B(\breve{x}, \breve{y})+\left(\sum_{\breve{s}=0}^{\breve{x}-1} \sum_{\breve{t}=0}^{\breve{y}-1} f(\breve{s}, \breve{t})\right)^{2}\right) \in \operatorname{Dom}\left(\breve{H}^{-1}\right) .
$$

\section{Applications}

The present section illustrates how Theorems 2.7 and 2.1 can be used to study the boundedness of the solutions of some initial boundary value problem for partial dynamic equations in two independent variables.

Let us consider the problem

$$
\begin{aligned}
& u^{\hat{\Delta} \hat{x} \hat{\Delta} \tilde{y}}(\breve{x}, \breve{y})=\breve{F}\left(\breve{x}, \breve{y}, u(\breve{x}, \breve{y}), \int_{0}^{\breve{x}} \breve{k}(\breve{s}, \breve{y}, u(s, \breve{y})) \hat{\Delta} \breve{s}\right), \\
& u(\breve{x}, 0)=a_{1}(\breve{x}), \quad u(0, \breve{y})=a_{2}(\breve{y}), \quad a_{1}(0)=a_{2}(0)=0,
\end{aligned}
$$

for any $(\breve{x}, \breve{y}) \in \Omega$, where $\breve{k} \in C_{\mathrm{rd}}(\Omega \times \mathbb{R}, \mathbb{R}), \breve{F} \in C_{\mathrm{rd}}(\Omega \times \mathbb{R} \times \mathbb{R}, \mathbb{R}), a_{1} \in C_{\mathrm{rd}}\left(\breve{\mathbb{T}}_{1}, \mathbb{R}\right)$ and $a_{2} \in C_{\mathrm{rd}}\left(\breve{\mathbb{T}}_{2}, \mathbb{R}\right)$.

Theorem 3.1 Suppose that the functions $\breve{k}, \breve{F}, a_{2}, a_{1}$ in (3.1) and (3.2) satisfy the conditions

$$
\begin{aligned}
& \mid \breve{F}(\breve{x}, \breve{y}, u(\breve{x}, \breve{y}, v) \mid \leq \bar{\varphi}(|u(\breve{x}, \breve{y})|)[f(\breve{x}, \breve{y}) \bar{\varphi}(|u(\breve{x}, \breve{y})|)+p(\breve{x}, \breve{y})] \\
&+f(\breve{x}, \breve{y}) \bar{\varphi}(|u(\breve{x}, \breve{y})|) v, \\
&|\breve{k}(\breve{x}, \breve{y}, u(\breve{x}, \breve{y}))| \leq g(\breve{x}, \breve{y}) \bar{\varphi}(|u(\breve{x}, \breve{y})|), \\
&\left|a_{1}(\breve{x})+a_{2}(\breve{y})\right| \leq a(\breve{x}, \breve{y}),
\end{aligned}
$$


where the functions $p, g, a, f$, and $\bar{\varphi}$ are defined as in Theorem 2.7 with $a(\breve{x}, \breve{y})>0$, for all $(\breve{x}, \breve{y}) \in \Omega$, then

$$
|u(\breve{x}, \breve{y})| \leq \breve{G}^{-1}\left(\breve{F}^{-1}\left[\breve{F}\left(q_{2}(\breve{x}, \breve{y})\right)+\int_{0}^{\breve{x}} \int_{0}^{\breve{y}} f(\breve{s}, \breve{t})\left[1+\int_{0}^{\breve{s}} g(\breve{\tau}, \breve{t}) \hat{\Delta} \breve{\tau}\right] \hat{\Delta} \breve{t} \hat{\Delta} \breve{s}\right]\right),
$$

for $0 \leq \breve{x} \leq \breve{x}_{1}, 0 \leq \breve{y} \leq \breve{y}_{1}$, where $F, q_{2}$ and $G$ are defined as in Theorem 2.7.

Proof If the problem (3.1) and (3.2) has a solution $u(\breve{x}, \breve{y})$, it can be written as

$$
u(\breve{x}, \breve{y})=a_{1}(\breve{x})+a_{2}(\breve{y})+\int_{0}^{\breve{x}} \int_{0}^{\breve{y}} \breve{F}\left(\breve{s}, \breve{t}, u(\breve{s}, \breve{t}), \int_{0}^{\breve{s}} \breve{k}(\breve{\tau}, \breve{t}, u(\breve{\tau}, t)) \hat{\Delta} \breve{\tau}\right) \hat{\Delta} \breve{t} \hat{\Delta} \breve{s}
$$

for any $(\breve{x}, \breve{y}) \in \Omega$. Using the conditions (3.3), (3.4) and (3.5) in (3.7), we get

$$
\begin{aligned}
|u(\breve{x}, \breve{y})| \leq & a(\breve{x}, \breve{y})+\int_{0}^{\breve{x}} \int_{0}^{\breve{y}} \bar{\varphi}(|u(\breve{s}, \breve{t})|)[f(\breve{s}, \breve{t}) \bar{\varphi}(\mid u(\breve{s}, \breve{t} \mid)+p(\breve{s}, \breve{t})] \hat{\Delta} \breve{t} \hat{\Delta} \breve{s} \\
& +\int_{0}^{\breve{x}} \int_{0}^{\breve{y}} f(s, t) \bar{\varphi}(|u(s, t)|)\left(\int_{0}^{\breve{s}} g(\breve{\tau}, \breve{t}) \bar{\varphi}(|u(\breve{\tau}, \breve{t})|) \hat{\Delta} \breve{\tau}\right) \hat{\Delta} \breve{t} \hat{\Delta} \breve{s},
\end{aligned}
$$

for any $(\breve{x}, \breve{y}) \in \Omega$. Now, an application of Theorem 2.7 to (3.8) yields the required inequality in (3.6) where $\bar{\psi}(u)=u$.

Let us consider the initial boundary value problem of the form

$$
\begin{aligned}
& \left(z^{q}\right)^{\hat{\Delta} \breve{x} \hat{\Delta} \breve{y}}(\breve{x}, \breve{y})=\breve{A}\left(\breve{x}, \breve{y}, z(\breve{x}, \breve{y}), \int_{0}^{\breve{x}} h(\breve{s}, \breve{y}, z(\breve{s}, \breve{y})) \hat{\Delta} \breve{s}\right) \\
& z(\breve{x}, 0)=a_{1}(\breve{x}), \quad z(0, \breve{y})=a_{2}(\breve{y}), \quad a_{1}(0)=a_{2}(0)=0,
\end{aligned}
$$

for any $(\breve{x}, \breve{y}) \in \Omega$.

Theorem 3.2 Assume that the functions $h, A, a_{2}, a_{1}$ in (3.9) and (3.10) satisfy the conditions

$$
\begin{aligned}
& \mid A\left(\breve{x}, \breve{y}, z(\breve{x}, \breve{y}, v)|\leq f(\breve{x}, \breve{y})| z^{r}(\breve{x}, \breve{y}) \mid+f(\breve{x}, \breve{y}) v,\right. \\
& |h(\breve{x}, \breve{y}, z(\breve{x}, \breve{y}))| \leq g(\breve{x}, \breve{y})\left|z^{r}(\breve{x}, \breve{y})\right|, \\
& \left|a_{1}(\breve{x})+a_{2}(\breve{y})\right| \leq a(\breve{x}, \breve{y})
\end{aligned}
$$

where $r \geq q>0$, then

$$
|z(\breve{x}, \breve{y})| \leq\left[(a(\breve{x}, \breve{y}))^{\frac{q-r}{q}}+\frac{q-r}{q} \int_{0}^{\breve{x}} \int_{0}^{\breve{y}} f(\breve{s}, \breve{t})\left(1+\int_{0}^{\breve{s}} g(\breve{\tau}, \breve{t}) \hat{\Delta} \breve{t}\right) \hat{\Delta} \breve{t} \hat{\Delta} \breve{s}\right]^{\frac{1}{q-r}},
$$

for $0 \leq \breve{x} \leq \breve{x}_{1}, 0 \leq \breve{y} \leq \breve{y}_{1}$.

Proof If the problem (3.9) and (3.10), has a solution $z(\breve{x}, \breve{y})$ it can be written as

$$
z^{q}(\breve{x}, \breve{y})=a_{1}(x)+a_{2}(y)+\int_{0}^{\breve{x}} \int_{0}^{\breve{y}} \breve{F}\left(\breve{s}, \breve{s t}, u(\breve{s}, \breve{t}), \int_{0}^{\breve{s}} \breve{k}(\breve{\tau}, \breve{t}, u(\breve{\tau}, \breve{t})) \hat{\Delta} \breve{\tau}\right) \hat{\Delta} \breve{t} \hat{\Delta} \breve{s}
$$


for any $(\breve{x}, \breve{y}) \in \Omega$. Using the conditions (3.11), (3.12) and (3.13) in (3.15), we get

$$
\begin{aligned}
\left|z^{q}(\breve{x}, \breve{y})\right| \leq & a(\breve{x}, \breve{y})+\int_{0}^{\breve{x}} \int_{0}^{\breve{y}} f(\breve{s}, \breve{t})\left|z^{r}(s, t)\right| \hat{\Delta} \breve{t} \hat{\Delta} \breve{s} \\
& +\int_{0}^{\breve{x}} \int_{0}^{\breve{y}} f(\breve{s}, \breve{t})\left(\int_{0}^{\breve{s}} g(\breve{\tau}, \breve{t})\left|z^{r}(\breve{\tau}, \breve{t})\right| \hat{\Delta} \breve{\tau}\right) \hat{\Delta} \breve{t} \hat{\Delta} \breve{s},
\end{aligned}
$$

from (3.16), we get

$$
\begin{aligned}
\left|z^{q}(\breve{x}, \breve{y})\right| \leq & a(\breve{x}, \breve{y})+\int_{0}^{\breve{x}} \int_{0}^{\breve{y}} f(\breve{s}, \breve{t})\left|z^{r}(\breve{t}, \breve{t})\right| \hat{\Delta} \breve{t} \hat{\Delta} \breve{s} \\
& +\int_{0}^{\breve{x}} \int_{0}^{\breve{y}} f(\breve{s}, \breve{t})\left(\int_{0}^{\breve{s}} g(\breve{\tau}, \breve{t})\left|z^{r}(\breve{\tau}, \breve{t})\right| \hat{\Delta} \breve{\tau}\right) \hat{\Delta} \breve{t} \hat{\Delta} \breve{s},
\end{aligned}
$$

for any $(\breve{x}, \breve{y}) \in \Omega$. A suitable application of Theorem 2.1 to (3.17) with $\bar{\psi}(u)=u^{q}, \bar{\varphi}(u)=u^{r}$ and $p(\breve{x}, \breve{y})=0$ gives the required inequality in (3.14).

\section{Acknowledgements}

We are immensely thankful to the editor and anonymous referees for their valuable remarks, which helped to improve the paper.

\section{Funding}

This research was funded by the Deanship of Scientific Research at Princess Nourah bint Abdulrahman University through the Fast-track Research Funding Program.

\section{Availability of data and materials}

Not applicable.

\section{Competing interests}

The authors announce that there are not any competing interests.

\section{Authors' contributions}

All authors have equally contributed to the manuscript, and read and approved it.

\section{Author details}

'Department of Mathematics, Faculty of Science, Al-Azhar University, Cairo, Egypt. ${ }^{2}$ Department of Mathematics, College of Science, Princess Noura bint Abdulrahman University, Riyadh, Saudi Arabia.

\section{Publisher's Note}

Springer Nature remains neutral with regard to jurisdictional claims in published maps and institutional affiliations.

Received: 26 October 2019 Accepted: 5 February 2020 Published online: 12 February 2020

\section{References}

1. Abdeldaim, A., El-Deeb, A.A.: Some new retarded nonlinear integral inequalities with iterated integrals and their applications in retarded differential equations and integral equations. J. Fract. Calc. Appl. 5(Suppl. 3S), Paper no. 9 (2014)

2. Abdeldaim, A., El-Deeb, A.A.: On generalized of certain retarded nonlinear integral inequalities and its applications in retarded integro-differential equations. Appl. Math. Comput. 256, 375-380 (2015)

3. Abdeldaim, A., El-Deeb, A.A.: On some generalizations of certain retarded nonlinear integral inequalities with iterated integrals and an application in retarded differential equation. J. Egypt. Math. Soc. 23(3), 470-475 (2015)

4. Abdeldaim, A., El-Deeb, A.A.: On some new nonlinear retarded integral inequalities with iterated integrals and their applications in integro-differential equations. Br. J. Math. Comput. Sci. 5(4), 479-491 (2015)

5. Abdeldaim, A., El-Deeb, A.A., Agarwal, P., El-Sennary, H.A.: On some dynamic inequalities of Steffensen type on time scales. Math. Methods Appl. Sci. 41(12), 4737-4753 (2018)

6. Agarwal, R., O'Regan, D., Saker, S.: Dynamic Inequalities on Time Scales. Springer, Cham (2014)

7. Agarwal, R.P., Lakshmikantham, V.: Uniqueness and Nonuniqueness Criteria for Ordinary Differential Equations. Series in Real Analysis, vol. 6. World Scientific Publishing, Singapore (1993)

8. Akin-Bohner, E., Bohner, M., Akin, F.: Pachpatte inequalities on time scales. JIPAM. J. Inequal. Pure Appl. Math. 6(1), Article ID 6 (2005)

9. Ammar, B.: On certain new nonlinear retarded integral inequalities in two independent variables and applications. Appl. Math. Comput. 2019(335), 103-111 (2018) 
10. Bohner, M., Guseinov, G.S.: Partial differentiation on time scales. Dyn. Syst. Appl. 13(3-4), 351-379 (2004)

11. Bohner, M., Guseinov, G.S.: Multiple integration on time scales. Dyn. Syst. Appl. 13(3-4), 579-606 (2005)

12. Bohner, M., Guseinov, G.S.: Double integral calculus of variations on time scales. Comput. Math. Appl. 54, 45-57 (2007)

13. Bohner, M., Matthews, T.: The Grüss inequality on time scales. Commun. Math. Anal. 3(1), 1-8 (2007)

14. Bohner, M., Matthews, T.: Ostrowski inequalities on time scales. JIPAM. J. Inequal. Pure Appl. Math. 9(1), Article ID 6 (2008)

15. Bohner, M., Peterson, A.: Dynamic Equations on Time Scales: An Introduction with Applications. Birkhauser, Boston (2001)

16. Dinu, C.: Hermite-Hadamard inequality on time scales. J. Inequal. Appl. 2008, Article ID 287947 (2008)

17. El-Deeb, A.A.: On Integral Inequalities and Their Applications. LAP Lambert Academic Publishing, Saarbrücken (2017)

18. El-Deeb, A.A.: Some Gronwall-Bellman type inequalities on time scales for Volterra-Fredholm dynamic integral equations. J. Egypt. Math. Soc. 26(1), 1-17 (2018)

19. El-Deeb, A.A.: A variety of nonlinear retarded integral inequalities of Gronwall type and their applications. In: Advances in Mathematical Inequalities and Applications, pp. 143-164. Springer, Berlin (2018)

20. El-Deeb, A.A.: On some generalizations of nonlinear dynamic inequalities on time scales and their applications. Appl. Anal. Discrete Math.

21. El-Deeb, A.A., Ahmed, R.G.: On some explicit bounds on certain retarded nonlinear integral inequalities with applications. Adv. Inequal. Appl. 2016, Article ID 15 (2016)

22. El-Deeb, A.A., Ahmed, R.G.: On some generalizations of certain nonlinear retarded integral inequalities for Volterra-Fredholm integral equations and their applications in delay differential equations. J. Egypt. Math. Soc. 25(3), 279-285 (2017)

23. El-Deeb, A.A., Cheung, W.-S.: A variety of dynamic inequalities on time scales with retardation. J. Nonlinear Sci. Appl. 11(10), 1185-1206 (2018)

24. El-Deeb, A.A., El-Sennary, H.A., Agarwal, P.: Some Opial-type inequalities with higher order delta derivatives on time scales. Rev. R. Acad. Cienc. Exactas Fís. Nat., Ser. A Mat. 114(1), 29 (2020)

25. El-Deeb, A.A., El-Sennary, H.A., Khan, Z.A.: Some Steffensen-type dynamic inequalities on time scales. Adv. Differ. Equ. 2019, 246 (2019)

26. El-Deeb, A.A., Elsennary, H.A., Cheung, W.-S.: Some reverse Hölder inequalities with Specht's ratio on time scales. J. Nonlinear Sci. Appl. 11(4), 444-455 (2018)

27. El-Deeb, A.A., Elsennary, H.A., Nwaeze, E.R.: Generalized weighted Ostrowski, trapezoid and Grüss type inequalities on time scales. Fasc. Math. 60, 123-144 (2018)

28. El-Deeb, A.A., Kh, F.M., Ismail, G.A.F., Khan, Z.A.: Weighted dynamic inequalities of Opial-type on time scales. Adv. Differ. Equ. 2019(1), 393 (2019)

29. El-Deeb, A.A., Xu, H., Abdeldaim, A., Wang, G.: Some dynamic inequalities on time scales and their applications. Adv. Differ. Equ. 130, 19 (2019)

30. El-Owaidy, H., Abdeldaim, A., El-Deeb, A.A.: On some new retarded nonlinear integral inequalities and their applications. Math. Sci. Lett. 3(3), 157 (2014)

31. El-Owaidy, H.M., Ragab, A.A., Eldeeb, A.A., Abuelela, W.M.K.: On some new nonlinear integral inequalities of Gronwall-Bellman type. Kyungpook Math. J. 54(4), 555-575 (2014)

32. Fan, M., Tian, Y., Meng, F.: A class of dynamic integral inequalities with mixed nonlinearities and their applications in partial dynamic systems. Adv. Differ. Equ. 12, 13 (2019)

33. Hilger, S.: Ein maßkettenkalkül mit anwendung auf zentrumsmannigfaltigkeiten. PhD thesis, Universität Würzburg (1988)

34. Hilscher, R.: A time scales version of a Wirtinger-type inequality and applications. J. Comput. Appl. Math. 141(1-2), 219-226 (2002)

35. Kh, F.M., El-Deeb, A.A., Abdeldaim, A., Khan, Z.A., et al.: On some generalizations of dynamic Opial-type inequalities on time scales. Adv. Differ. Equ. 2019(1), 1 (2019)

36. Li, J.D.: Opial-type integral inequalities involving several higher order derivatives. J. Math. Anal. Appl. 167(1), 98-110 (1992)

37. Li, W.N. Some delay integral inequalities on time scales. Comput. Math. Appl. 59(6), 1929-1936 (2010)

38. Řehák, P.: Hardy inequality on time scales and its application to half-linear dynamic equations. J. Inequal. Appl. 5, 495-507 (2005)

39. Saker, S.H., El-Deeb, A.A., Rezk, H.M., Agarwal, R.P.: On Hilbert's inequality on time scales. Appl. Anal. Discrete Math. 11(2), 399-423 (2017)

40. Tian, Y., El-Deeb, A.A., Meng, F.: Some nonlinear delay Volterra-Fredholm type dynamic integral inequalities on time scales. Discrete Dyn. Nat. Soc. 2018, Article ID 5841985 (2018)

41. Tian, Y., Fan, M., Meng, F.: A generalization of retarded integral inequalities in two independent variables and their applications. Appl. Math. Comput. 221, 239-248 (2013) 